\title{
3D model discretization in assessing urban solar potential: the effect of grid spacing on predicted solar irradiation
}

\author{
Giuseppe Peronato ${ }^{\mathrm{a}}$, Emmanuel Rey ${ }^{\mathrm{b}}$, Marilyne Andersen ${ }^{\mathrm{a}}$ \\ ${ }^{a}$ Laboratory of Integrated Performance in Design (LIPID) \\ ${ }^{b}$ Laboratory of Architecture and Sustainable Technologies (LAST) \\ Ecole polytechnique fédérale de Lausanne (EPFL) \\ Lausanne (Switzerland)
}

\begin{abstract}
The increasing interest in solar energy production in urban areas requires an accurate simulation of solar irradiation on building surfaces, including vertical surfaces. However, solar potential analyses are usually conducted on 2.5D models, which are limited to roof surfaces. Methods based on 3D models, instead, allow the simulation of solar radiation on all building surfaces also accounting for inter-reflections. 3D models are thus discretized by grids of sensor points on which the solar potential is calculated.

This paper investigates the discretization error in the assessment of solar potential based on 3D models. To this end, we tested the sensitivity of simulated solar irradiation to the resolution of the sensor grid. We analyzed the impact of the grid resolution using typical discretization approaches affecting the spatial arrangement of the sensor points.

The study was conducted in a dense area of the city of Geneva represented at level of detail (LOD) 2. The simulated solar irradiation on 109 buildings was analyzed at different spatial, i.e. per surface and per building, and temporal granularities, i.e. hourly and yearly.

The results show that the error increases linearly for grids spaced at up to $4 \mathrm{~m}$ with maximum relative root mean square error lower than $7 \%$. The impact of the grid resolution was found greater for structured grids than unstructured grids. The results also highlight that finer grid resolutions (i.e. smaller spacing) are necessary if the analysis is conducted at high spatial or temporal granularity, notably when analyzing roof surfaces with shading artifacts.
\end{abstract}

Keywords: Solar potential, 3D city model, Mesh generation, Uncertainty analysis 


\section{Introduction}

Since buildings are the largest energy-consuming sector in the world (IEA, 2013, p. 1), there is a growing interest towards solar energy in urban environments, so as to provide decentralized energy production where it is most needed. Some countries and local governments require solar energy systems to be installed in new buildings. However, new buildings represent usually only a small share of the entire building stock. For this reason, the installation of solar energy systems in existing buildings is crucial.

On the basis of the building-integrated photovoltaic (BIPV) potential calculated by the IEA (2002), it can be estimated that solar systems installed on building surfaces could cover about one third of the energy consumption for many industrialized countries. Moreover, BIPV installations can improve the economic and energy viability of building renovations (Aguacil Moreno et al., 2016).

About $15-20 \%$ of the total BIPV potential would be possible thanks to solar systems integrated into façades (IEA, 2002), whose potential should not be neglected. Despite providing a lower annual solar yield than rooftop-applied systems due to the unfavorable tilt angle and shading conditions, façade-installed systems can help smooth the seasonal and daily production curves and improve the match with the building energy demand (Sánchez \& Izard, 2015; Aguacil Moreno et al., 2016). On the other hand, we can assume that the effect of urban shading and albedo have a significant impact on façades' solar irradiation. For these reasons, adequate simulation methods must be applied. However, most of the existing methods are based on image-processing of Digital Surface Models (DSM) derived from aerial surveys (e.g. LiDAR data). Because of the 2.5D nature of such models, they consider only roof surfaces and do not take into account reflected solar radiation ${ }^{1}$. Recent implementations have extended this analysis method also to vertical surfaces, using pixels from 3D textures as sensor points and voxel grids as occlusion geometry (Bremer et al., 2016) or using sensor points placed at different height intervals derived from the 2.5D model (Catita et al., 2014; Brito et al., 2017). However, the former method does not account for the effect of inter-reflections, while the latter not even of discontinuous vertical surfaces, such as façades covered by over-hangs.

In order to include a complete simulation of façades accounting for the effect of overhangs and inter-reflections, we implemented a method based on 3D geometry and solar radiation simulation tools using backwards ray-tracing, similar to the one first developed by Compagnon \& Raydan (2000). This method requires as input

\footnotetext{
${ }^{1}$ For a complete state-of-the-art review see for example Freitas et al. (2015)
} 
some sensor points ${ }^{2}$, i.e. oriented $3 \mathrm{D}$ points to which the solar radiation model is applied. The sensor points are created using an algorithm subdividing the surfaces to be analyzed and forming hence several sensor grids.

Image-based analysis based on pixel discretization for a given view (Mardaljevic \& Rylatt, 2000), rather than on discretization of the analyzed surfaces, have also been applied to urban solar potential assessments. However, as can be seen in Table 1, sensor-point-based methods have become widespread and it is thus worth analyzing their discretization techniques.

This work addresses the question of the most appropriate grid characteristics for urban-scale solar potential assessments. In particular, it focuses on the grid resolution (i.e. the spacing between the sensor points) and spatial arrangement (i.e. whether sensor points are distributed according to a regular and constant spacing interval - structured grid - or as the faces of the mesh from which they are derived unstructured grid).

The computational cost of the simulations being correlated to the number of sensor points, a lower resolution (i.e. greater spacing) grid is beneficial in terms of simulation time, but an acceptable accuracy should be guaranteed and the confidence intervals be known. Therefore, this work aims to quantify the error that has to be considered when using grid resolutions lower than $0.5 \mathrm{~m}$, which we assumed to be the ground-truth. We also wanted to check whether the use of a structured or unstructured grid affects the simulated solar irradiation, as each grid-creation method presents a different spatial arrangement and quantity of sensor points.

In order to define some recommendations for solar potential analyses, we finally tried to answer the following questions while considering the specificity of the case study in an urban context:

1. At which resolution does the discretization error become acceptable?

2. Is solar irradiation over- or under-estimated when using low-resolution grids?

3. In which spatial and temporal conditions does the influence of grid resolution affect the results the most?

\section{State of the art}

This section reviews previous work dealing with sensitivity of solar radiation to different input data, parameters and models as well as the consequent uncertainty (§2.1). It also reports the common practice for the discretization of 3D models, in

\footnotetext{
${ }^{2}$ In the literature, we find equivalent alternative terms, such as for example "sensor nodes", "test points", "probe points", "query points".
} 
terms of resolution and spatial arrangement of sensor grids, and the reasons behind 70 the use of particular settings $(\S 2.2)$. We finally summarize in $\S 2.3$ these findings indicating what is currently missing with regards to the scope of this paper.

\subsection{Uncertainty analysis in solar potential assessments}

Uncertainty analysis is primordial when dealing with models, as in simulationbased assessments. Uncertainties can be defined as "potential deficiencies due the lack of knowledge" (AIAA as cited in Iaccarino, 2009). We can distinguish from aleatory uncertainty, which is intrinsic to the nature of the data and cannot be reduced, and epistemological uncertainty, which implies a nominal lack of knowledge due to the methods used (ibid.). The latter could potentially be reduced, but usually at a cost (e.g. better instrument or longer computation time).

Unacknowledged errors, such as the ones due to the applications of models or algorithms, can be also considered uncertainties because they are caused by a lack of knowledge (AIAA as cited in Iaccarino, 2009). Uncertainty analysis is used to test the model or input data against reference models or high-resolution data in order to retrieve the associated error.

The simulation of solar irradiation is based on different input data and mathematical models. Input data typically include a 3D representation of obstructions (e.g. buildings, trees, terrain), their material properties (e.g. transparency, reflectance), and measured or statistically-sampled irradiance values. Models are first applied for calculating irradiance values on tilted surfaces from horizontal and normal values, which are usually provided by meteorological stations. Prada et al. (2014) showed that, in the context of building energy performance, the application of different radiation models produce a dispersion of simulation outcomes, hence an uncertainty in the predicted energy performance.

Other uncertainties occur with the models accounting for the shading and reflection from the context. In this case, the choice is often limited when the analysis target is the urban or regional scale. Models developed for large-scale applications (for example Š́ri \& Hofierka, 2004; Robinson \& Stone, 2004a,b) typically rely on different simplifications of the physical reality to reduce the simulation time. For instance, the method by Śúri \& Hofierka (2004), which is conceived for regionalscale applications in a GIS environment, presents several simplifications in terms of reflected radiation and weather variability; the method by Robinson \& Stone (2004a), while being based on physically-accurate backwards ray-tracing, implements sky patches accounting for both direct and diffuse contribution to simulate irradiation values cumulated over a certain period of time; Robinson \& Stone (2004b) implemented instead a simplified radiosity algorithm (SRA) to include the contribu- 
tion of reflected solar radiation by neighbor obstructions. All these methods have been tested against a reference model, which is assumed to be physically accurate, or measured values. Robinson \& Stone (2004a,b) used irradiance data simulated with Radiance sub-program gendaylit on an urban canyon, while Šúri \& Hofierka (2004) used measured horizontal global irradiation from a database.

Even if detailed models are available, the choice of coarse discretization settings can help reduce the simulation time, at the cost of loss in accuracy though. In this sense, some studies focused on the error due to the use of low resolutions for sky subdivision and simulation time step (Alam et al., 2016) and resolution of 3D models (Alam et al., 2016; Bremer et al., 2016). The aspects related to geometry discretization will be further analyzed in the following section.

In some cases, an error has to be accounted due to the difficulty of obtaining accurate input data. 3D models at high level of detail (LOD) are often not available for large urban areas, as they are based on expensive aerial surveying and often manual corrections, while in some cases they are possibly not the best choice for some specific applications, because they cause longer simulation time without significantly improving the results (Biljecki et al., 2015b). In this sense, Besuievsky et al. (2014) and Peronato et al. (2016a) investigated the effect of using 3D models with coarser LODs. Similarly, but with an application on simulation of building energy demand, previous work analyzed the effect of LOD (Strzalka et al., 2015; Nouvel et al., 2017), semantic data quality (Nouvel et al., 2017) and spatial accuracy (Wate et al., 2016) on the simulation results. Biljecki et al. (2015a) investigated the propagation of measurement errors in 3D models at different LODs on the calculated solar irradiation.

Others instead focused on the uncertainty due to parameters whose physical variability is not always practical or cost-effective to model at the urban scale, such as vegetation (Fogl \& Moudrý, 2016; Bremer et al., 2016; Peronato et al., 2016b), or which are dependent on user choices, like for the geometric regularity in the arrangement of PV modules (Peronato et al., 2015).

The above-cited work use different methods on which the uncertainty can be assessed, depending on the model and data input. Uncertainty is thus defined:

- with reference to a ground-truth, which can be either measured (Š́ri \& Hofierka, 2004) or assumed to represent more accurate results (Besuievsky et al., 2014; Peronato et al., 2016a; Strzalka et al., 2015; Nouvel et al., 2017; Robinson \& Stone, 2004a,b);

- with extreme scenarios, whose simulation results are supposed to represent the confidence intervals (Peronato et al., 2015, 2016b); 
- with a probabilistic approach, to define confidence intervals out of aleatory uncertainty such as the one represented by weather (Rastogi, 2016) and positional error in 3D data (Biljecki et al., 2015a; Wate et al., 2016).

\subsection{Geometry discretization}

Sensor grids are used as input to simulation models for different uses, including noise propagation (Stoter et al., 2008), solar radiation simulations (Freitas et al., 2015; Bremer et al., 2016), visibility assessments (Florio et al., 2016) and computational fluid dynamics (Chung, 2002, part four). The grids are produced by sampling 3D city models with 3D point-clouds using different discretization algorithms.

Unlike the level of detail (LOD), whose choice is usually limited by the available data and the type of analysis, the grid discretization depends on the scope and the target accuracy of the analysis. The finer the grid is, the more accurate the results are expected to be, at the cost though of longer simulation time. In the case of arrangement, the choice is mostly dictated by the type of assessment and expected output.

Based on the terminology derived from computational fluid dynamics (CFD), we can distinguish between two types of arrangement:

- structured grids, in which "it is possible to define a curvilinear co-ordinate system that spans over the entire domain and hence the connectivity of the individual sub-domains is implicit"( (Niyogi et al., 2006, 8.1);

- unstructured grids, in which "the connectivity of the individual sub-domains must be explicitly specified" (ibid.).

In CFD, both structured and unstructured grid arrangements are used, with the latter being usually preferred for complex geometry, in particular in the form of adaptive mesh resolution to maximize efficiency and accuracy (Chung, 2002, part four).

With regards to solar energy assessments, different discretization settings for grid spacing and arrangement are used in the literature. However, the choice is often not justified or is only based on reference values from the literature (in particular, Compagnon, 2004). Moreover, as can be seen in Table 1, there is no correlation between the LOD and the grid size, while intuitively the higher the LOD, the lower the grid spacing should be. Depending on the data input and modeling platform, methods based on either a structured or unstructured grid are used in the literature, without their choice being usually justified either by supporting arguments or by the specific analysis scope. There are though two exceptions. Waibel et al. (2016) 
motivated the choice of (unstructured) meshing algorithms arguing that the density of the sensor points increases with the complexity of the geometry. However, it can be also argued that complex radiation and shading patterns can affect simple rectangular surfaces, for instance a façade in an urban canyon. Peronato et al. (2015) used instead a regular grid as their purpose was to include a geometric regularity criterion in the assessment of the BIPV potential.

Table 1: Typical grid spacing and arrangements, level of detail (LOD) and scale of analysis of the 3D models. Analysis focusing on single buildings are not included.

\begin{tabular}{ccccc}
\hline Reference & Grid spacing & LOD & Grid arrangement & Scale \\
\hline Compagnon \& Raydan (2000) & $0.5-1 \mathrm{~m}$ & LOD1 & Structured & Neighborhood \\
Compagnon (2004) & $1 \mathrm{~m}$ & LOD1 & Structured & Neighborhood \\
Kämpf (2009) & $1^{3} \mathrm{~m}$ & LOD2 & Unstructured & Neighborhood \\
Montavon (2010) & $1 \mathrm{~m}$ & LOD1 & Structured & Neighborhood \\
Nault et al. (2013) & $4 \mathrm{~m}$ & LOD1 & Unstructured & Neighborhood \\
Jakubiec \& Reinhart (2013) & $1.5 \mathrm{~m}$ & LOD2 & Unstructured & Urban \\
Peronato (2014) & $1 \mathrm{~m}$ & LOD1 & Unstructured & Neighborhood \\
Catita et al. (2014) & $0.5 \mathrm{~m}$ & LOD3 & Structured & Neighborhood \\
Wieland et al. (2015) & $2^{3} \mathrm{~m}$ & LOD2 & Structured & Neighborhood \\
Fath et al. (2015) & $1.5 \mathrm{~m}$ & LOD2 & Unstructured & Urban \\
Waibel et al. (2016) & $2.1-4.3^{4} \mathrm{~m}$ & LOD1 & Unstructured & Neighborhood \\
Peronato et al. (2016b) & $1 \mathrm{~m}$ & LOD1 & Structured & Urban \\
Nault et al. (2017) & $1 \mathrm{~m}$ & LOD1 & Unstructured & Neighborhood \\
Costanzo et al. (2018) & $1.5 \mathrm{~m}$ & LOD1 & Unstructured & Neighborhood \\
Vulkan et al. (2018) & $1 \mathrm{~m}$ & LOD1 & Structured & Neighborhood \\
\hline
\end{tabular}

Yet, to the best of authors' knowledge, the influence of the sensor grid resolution and type has been mostly neglected in precedent studies. Only Alam et al. (2016) and Bremer et al. (2016) focused on this aspect, though using simplified solar potential calculations, neglecting for example reflected radiation.

Kämpf (2009, p.11) also run a sensitivity analysis using a decomposition algorithm into equiareal sub-surfaces (which we can consider as unstructured), while not explicitly mentioning the optimal value found using Radiance simulations.

Alam et al. (2016) checked the sensitivity of the sky view factor to the meshing resolution of buildings, using different triangulation areas ranging from $5 \mathrm{~m}^{2}$ to 0.01 $\mathrm{m}^{2}$. They showed a significant influence only on shaded areas, for which the solar

\footnotetext{
${ }^{3}$ Information retrieved from the authors, as not specified in the published paper.

${ }^{4}$ Mininimum-maximum values, as mesh size varies during the optimization process. Information retrieved from the authors, as not specified in the published paper.
} 
radiation is generally low. However, they did not come up with an optimal resolution for this kind of assessments, but rather stated that the quality of the meshing resolution is proportional to the accuracy of the result. Moreover, their method did not include inter-reflections nor the assessment of a structured grid.

Conversely, Bremer et al. (2016) only analyzed a structured sensor grid and did not discussed unstructured grid arrangements. This sensor grid was in fact created using a texture-mapping algorithm, which necessarily works with regular grids as based on pixels, and was proved to provide a powerful visualization and an efficient data storage. They also implemented a structured voxel grid for representing occlusions in their radiation model. They hence analyzed the impact of different resolutions $(0.5,1.0,2.5,5.0$ and $10.0 \mathrm{~m})$ of sensor grids, as well as of the occlusion voxels. They showed that the resolution of the sensor grid has a greater impact than the resolution of the occlusion geometry and significantly affects the accuracy of the results. Even if they applied a 1-m resolution for their case-study application, they did not suggest an optimal resolution, but rather showed the potential of using a multi-scale method for improving the computational performance.

\subsection{Summary of findings}

We have shown that many studies analyzed the sensitivity of building performance and solar potential to different models and input data. These studies provide information on the uncertainty that has to be considered, expressed for instance as a confidence interval. For urban-scale assessments, in most of the cases the evaluation of the assessment is done with regards to a reference model or high-resolution input data.

With the exception of the work by Kämpf (2009), Bremer et al. (2016) and Alam et al. (2016) but within their previously-exposed limitations, we have not found other previous studies investigating the effect of 3D geometry discretization in solar potential assessments. On the contrary, standard values are usually chosen with reference to previously-published work. However, we argue that the choice of both grid type and resolution should be justified with regards to their actual impact on simulation results, similarly to what has been already done by the cited studies addressing the topic of uncertainty on predicted solar irradiation.

\section{Methodology}

The methodology of this work is composed of two main phases. The first phase consists in a simulation-based workflow (which is illustrated in Figure 1) to analyze the effect of different discretization settings on solar irradiation. This workflow is 
tested in an urban area of the city of Geneva, which will be presented in $\S 3.1$. The second phase is based on a similar workflow, but it is applied to a single building using more accurate simulations (i.e. using the actual sun positions instead of a daylight coefficient method) in order to check the accuracy of the previous results. The main steps of the first phase will be further explained in $\S 3.2, \S 3.3, \S 3.4, \S 3.5$, while the second phase will be detailed in $§ 3.6$.

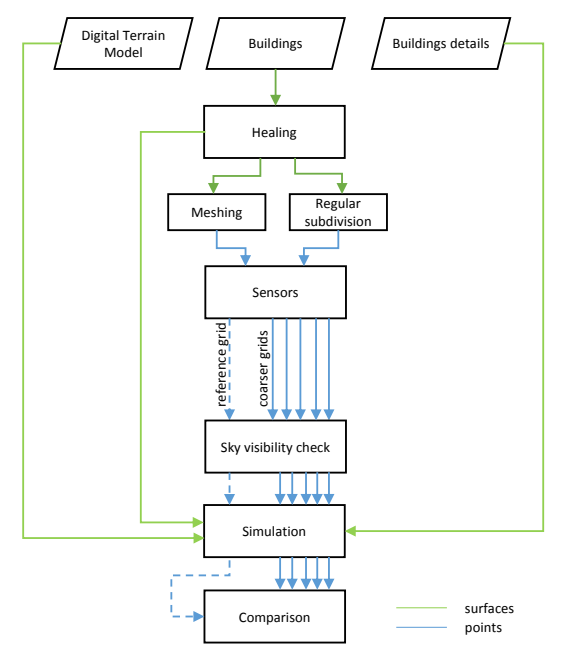

Figure 1: Schematic of the analysis workflow

235

The core of the analysis is the comparison of solar irradiation values calculated with different discretization settings. We implemented a parametric analysis on 5 different resolution levels $(0.5,1,2,3$ and $4 \mathrm{~m})$ and 3 grid creation algorithms (Unstructured grid, Structured grid, Surface center point). Since the Surface center point algorithm is independent on resolution, we thus studied in total $5 \cdot 2+1=11$ discretization settings.

In order to define the discretization error, we used the reference error with regards to the first resolution level, i.e. the grid with a spacing interval of $0.5 \mathrm{~m}$, which provides the maximum number of sensor points for each surface. Unlike for the sensor grid resolution ${ }^{3}$, we could not define a reference arrangement to be considered as the ground-truth. While the number of sensor points is greater for an unstructured grid, as can be seen in Fig. 4a, their arrangement is not homogeneous and might

\footnotetext{
${ }^{3}$ The terms "grid size", "grid spacing" and "grid resolution" are used used as interchangeable synonyms in this work.
} 
hence over-estimate the contribution of sensor points at the edges of the analyzed surface. For this reason, we did not focus on the effect of different grid arrangement types, but only on how results from a particular grid arrangement (structured or unstructured) are affected by the grid resolution.

Tools. The handing and healing of the geometry is done in Grasshopper ${ }^{4}$, an algorithmic environment for Rhinoceros $3 \mathrm{D}^{5}$, which provides powerful libraries for managing and editing 3D models. Simulations are conducted in Daysim ${ }^{6}$, a validated irradiance/illuminance simulation engine (Reinhart \& Walkenhorst, 2001) based on Radiance $^{7}$, a physically-accurate backwards ray-tracer (Ward-Larson \& Shakespeare, 1998), using Honeybee as the graphical interface (Roudsari \& Pak, 2013). Data analysis, i.e. the comparison of simulation results using different grids, is conducted using Python scripting, while relying on Rhino-Grasshopper for the visualization of spatial false-color maps.

\subsection{Case study}

The studies evaluating the quality of 3D models in environmental simulations are typically based on data created by either procedural modeling (Besuievsky et al., 2014; Biljecki et al., 2015a, 2017) or extracted from datasets of existing cities (Strzalka et al., 2015; Nouvel et al., 2017; Peronato et al., 2016b). However, procedural deling engines cannot easily reproduce the variety of building morphologies and configurations typical of many historical cities. Case studies of real urban areas represent hence a valid alternative method, provided that the level of detail (LOD) of their 3D model can capture as much as possible of their diversity. For example, roof superstructures from a real city are often of very different shapes and positions and are hence expected to produce a variable effect on solar irradiation, at least when the analysis is conducted at adequate granularity.

The urban area we used in this study is a 350x350-m tile in the center of the city of Geneva. The tile includes 322 buildings, of which 109 fall within the analyzed inner urban area, while the others are part of a buffer zone of $50 \mathrm{~m}$ which is taken into account only as obstructing geometry. The Floor Area Ratio (FAR), which is a standard metric for urban measuring built density, of the selected tile, including the buffer zone of $50 \mathrm{~m}$, is $\mathbf{3 . 1}$ and the median building height is $\mathbf{1 2} \mathbf{~ m}^{8}$.

\footnotetext{
${ }^{4}$ Version 0.76 (http://www.grasshopper3d.com/, last accessed on February 2017)

${ }^{5}$ Version 5.12 64-bit (http: //www.rhino3d.com/ last accessed on February 2017)

${ }^{6}$ Version 4.0 (http://www. daysim.com/, last accessed on February 2017)

${ }^{7}$ Version 5.0 (http://www.radiance-online.org/, last accessed on February 2017)

${ }^{8}$ The floor area value for the FAR is calculated by considering the average building height and
} 
This tile includes a good variety of buildings shapes and sizes, while presenting a density value which is common for many inner areas of European and NorthAmerican cities. In terms of roof superstructures, the tile features flat, gable and hip roofs, as well as various types of architectural details (dormers, HVAC systems). In this sense, the 3D model of the Canton of Geneva at LOD $2.3{ }^{9}$ provides a detailed representation of buildings, including their wide range of superstructures. Moreover, with the exception of a church, no building is enlisted in the heritage protection list, making the installation of BIPV systems possible, at least from a legal point of view.

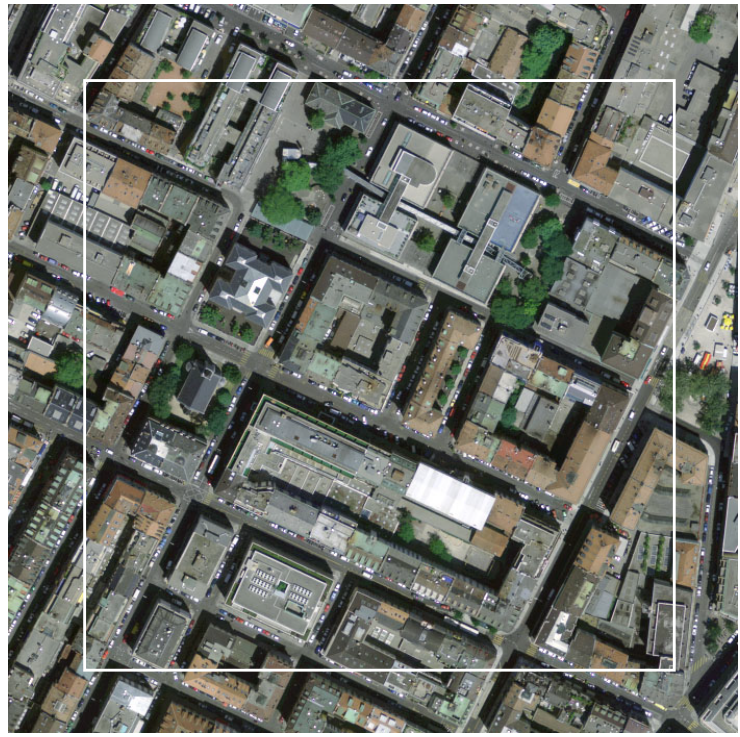

(a) Orthophoto

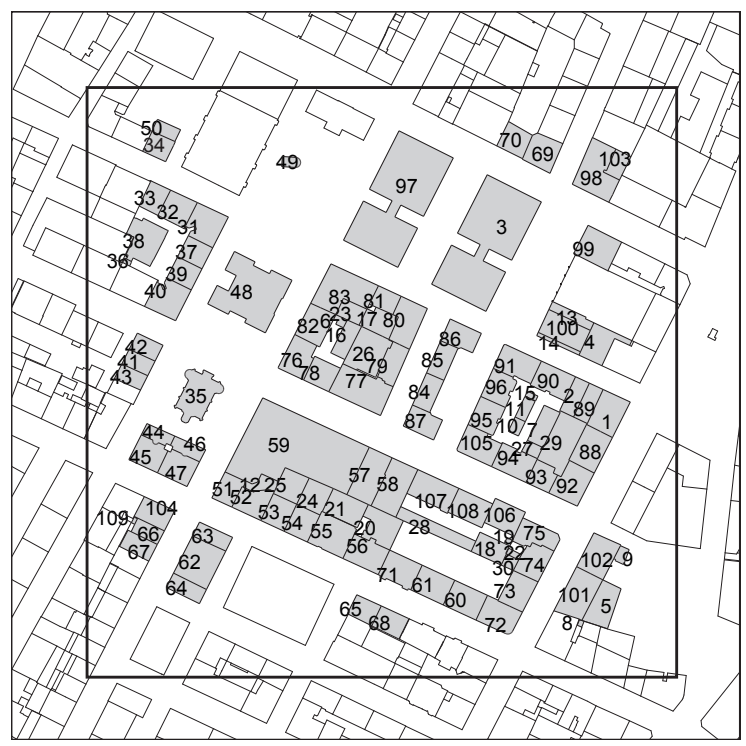

(b) Plan

Figure 2: The analyzed buildings are located within the inner square and are tagged with their ID in $2 \mathrm{~b}$.

\subsection{Importing and healing of the geometry}

The geodata, composed of a 3D model of buildings and a Digital Terrain Model at $0.5-\mathrm{m}$ resolution, are first trimmed in ArcGIS and exported respectively as *.SHP and *.TIF. These files are then converted into *.DXF and *.XYZ files, using $\mathrm{GDAL}^{10}$, specifically the ogr2ogr and gdal_translate libraries. These files are hence

assuming a constant floor height of $3 \mathrm{~m}$, while the plot area is considered as the entire surface of the 350x350 m tile, including hence both private plots and public space.

${ }^{9}$ For the LOD X.Y classification see Biljecki et al. (2014).

${ }^{10}$ Version 2.1.0, 2016/04/25 (http://www.gdal.org/, last accessed on March 2017) 
imported in Rhino-Grasshopper respectively as points and curves. The point cloud is directly converted to a mesh, through a Delaunay triangulation. The curves of building geometry are subjected to the following healing procedures, to ensure that the geometry is topologically correct and appropriate for the simulation.

Planarity. We tested the curves imported from the DXF files for planarity. The vertices of the curves not passing the test are projected to the best-fitting plane. New planar curves can be created out of the projected points and all curves are thus converted to surfaces.

Surface orientation. We adopted a simulation-based approach to check whether the building surfaces are correctly oriented, i.e. outwards with respect to the center of the building. To this end, a simple Radiance simulation with an uniform sky was run on sensor points created with a meshing algorithm at a $10-\mathrm{m}$ resolution. We considered as reversed surfaces those having more than $95 \%$ sensor points with less than $5 \%$ of the maximum illuminance value obtained in the area. Unfortunately, this procedure fails to reverse some inwards-oriented surfaces, such as those that are partially adjacent to other surfaces, because the ratio of their sensor points than can see the sky is usually below the set threshold (95\%). Even if these surfaces (yellowcolored. in Fig. 4) cannot be included in the simulations, we assume that this does not affect the assessment process, as it is unlikely that such surfaces will be used for installing BIPV systems.

\subsection{Creation of the sensor points}

We implemented two different techniques for creating irradiance sensor points, based on the existing algorithm and a custom-made workflow. In both cases the output is a set of $3 \mathrm{D}$ points and vectors which are used as input for the simulation. 315 The two algorithms produce a much different quantity (Fig. 3a) and distribution (Fig. 4) of sensor points on surfaces.

Unstructured grid. The Generate test points ${ }^{11}$ component included in Honeybee (Roudsari \& Pak, 2013) was used to create the sensor points. This component first creates a mesh with a target minimum and maximum face edge length which is equal to the set spacing interval. However, the density of mesh faces is not constant and, as can be seen in Fig. 3a, is generally higher close to the polygon edges, but it allows a complete coverage of buildings surfaces. The center point of each mesh face and

\footnotetext{
${ }^{11}$ Version 0.0.59, January 26, 2016
} 
the corresponding normal vectors are then used as sensor points for the simulation, as done by previous studies (e.g. Alam et al., 2016).

Structured grid. With the exception of the dxgridmaker program included in the STADIC tool (Casey \& Mistrick, 2015), which is, however, limited to horizontal surfaces, no out-of-the-box solutions exist for creating a structured and evenly-spaced sensor grid adapted for simulations in the Radiance/Daysim platform. The grid is hence produced using a custom Grasshopper workflow based on the one developed by Peronato et al. (2015) and adapted to keep the spacing interval constant across different surfaces. In fact, because of the division in equal segments conducted with the Divide Surface Grasshopper component, the spacing between each point is constant within one surface and direction but not always correspondent to the target spacing interval. For this reason, we introduced here two further steps to ensure a constant distance interval between the sensor points. The entire workflow consists of the following steps:

1. All surfaces are rebuilt while rotated along the $\mathrm{x}, \mathrm{y}$ axis, so that their $\mathrm{u}-\mathrm{v}$ axes are oriented accordingly:

2. Each surface is divided in equal segments along the $u-v$ axes of the surfaces:

3. The point grids of each surface are scaled up so that the distance between each point corresponds to the target spacing interval;

4. Points that got off the corresponding surface because of the scaling procedure are discarded. It should be noted that this algorithm determines that the sensor points are centered on the corresponding façade, i.e. with an offset with respect to its boundaries.

It should be noted that this algorithm determines an offset of the sensor points with regards to the boundaries of the underlying surfaces. This offset augments along with the decrease of the resolution. The implications of this arrangement will be further discussed in $§ 5.2$.

Surface center point. In this special case, a single sensor point is fixed in the geometric center of each surface. Considering that in our 3D model a surface usually corresponds to a semantic surface (e.g. a wall, a roof face), this grid arrangement provides the least defined grid (e.g. composed by a single point), while it is supposed to represent the average behavior of that surface considering that is located in its center. For this reason, this method is commonly used for simulations of solar irradiation intended for the assessment of building thermal performance. 


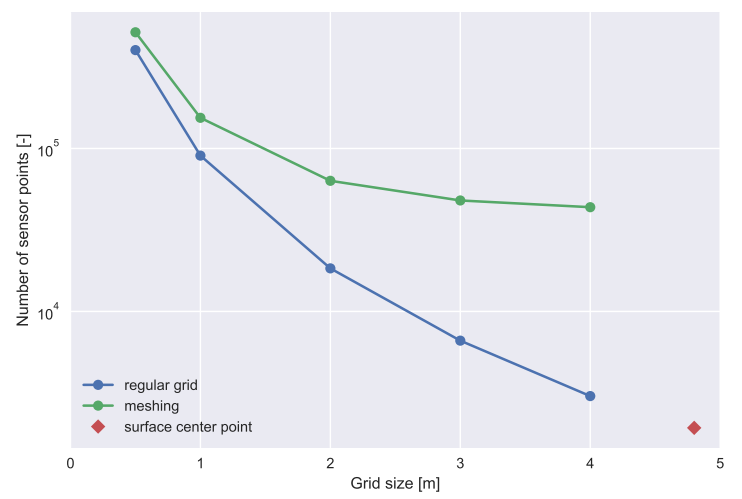

(a)

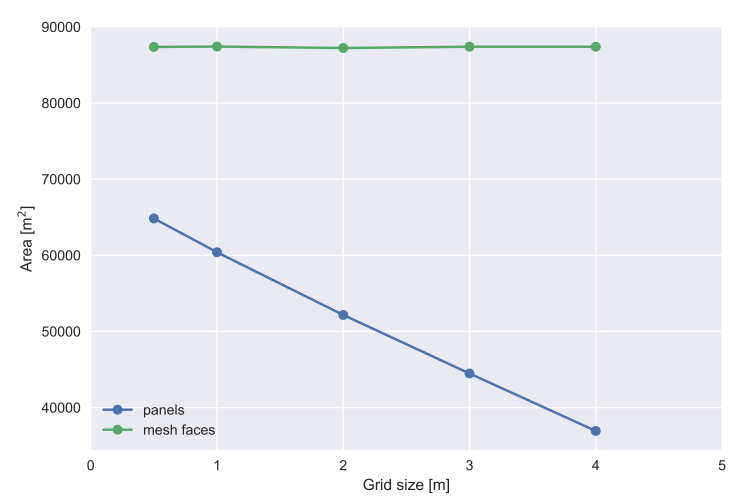

(b)

Figure 3: Characteristics of the two grid types in terms of number of sensor points (a) and mesh face area (b). The number of sensor points when placed at the surfaces' centers (red marker) has been arbitrarily plotted with $x=480$. Only surfaces with at least one sensor at $4-\mathrm{m}$ spacing are considered. As the spacing interval (grid size) increases, we observe a much greater decrease in the number of sensor points than for the mesh points.

In order to reduce the calculation time, we also checked whether each point actually see the sky. This is done trough a Radiance simulation using a constant diffuse sky. Only points seeing the sky have been included in the simulation. It should be noted that roof superstructures such as dormers were not included in this check. For this reason, points distributed on a roof surface, in reality might be covered by a dormer or other superstructures. This was chosen in order to assure the comparability of results (i.e. the same grid) with 3D models at LOD2, which do not include such details.

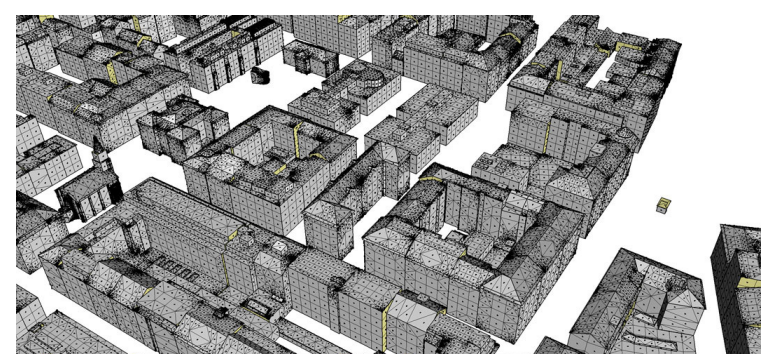

(a)

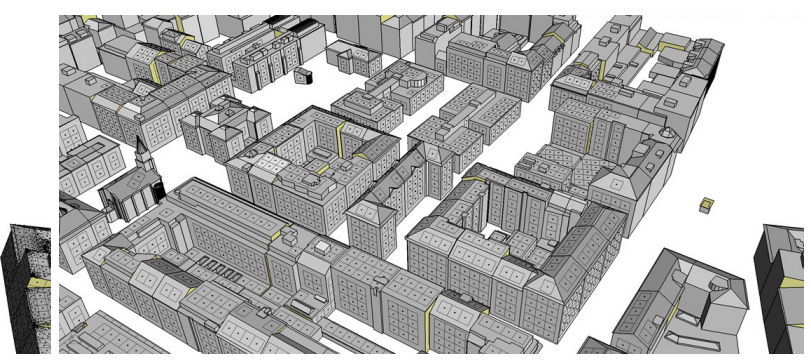

(b)

Figure 4: Arrangement of sensor points with the two types of discretization algorithms: at left, unstructured grid (mesh), at right, structured grid. The algorithms generating these grids are described in $\S 3.3$. 


\subsection{Simulation}

The simulation inputs are the sensor grid (point and normals coordinates), the geometry (buildings and terrain meshes), the material definitions and the weather files.

Building surfaces and terrain are defined as Lambertian diffusers with 0.30 and 0.10 reflectivity respectively, as suggested by IESNA (2012).

Concerning ray tracing, the Radiance parameters are listed in Table 2. In particular, the number of admissible ambient bounces (-ab parameter) is set to 2 , which means that during the ray-tracing, one reflection of each ray from the context is taken into account. This is considered an acceptable simplification for urban-scale simulations. However, the influence of these parameters will be checked in a sensitivity analysis $(\S 4)$

We used a weather file containing data of a typical meteorological year (TMY) for Geneva made by ASHRAE IWEC and available from EnergyPlus website ${ }^{12}$.

Table 2: Radiance parameters used in this work: ambient bounces - $a b$, ambient resolution - $a b$, ambient divisions - $a d$, ambient super-samples - $a s$, ambient accuracy - $a a$.. Other settings for $-a b$ and -ar parameters have been tested in $\S 4$.

\begin{tabular}{ccccc}
\hline$-a b$ & $-a r$ & $-a d$ & $-a s$ & $-a a$ \\
\hline 2 & 300 & 1000 & 500 & 0.1 \\
\hline
\end{tabular}

\subsection{Analysis metrics}

Using the simulation output (hourly irradiance value for each sensor node), the yearly solar irradiation of each surface normalized by surface area was computed. We then obtained the suitable surface and irradiation as a function of different irradiation thresholds using the following equations:

$$
\begin{array}{ll}
\text { SuitArea }=\sum_{s=1}^{n} A_{s} \cdot t_{s} & \text { with } t_{s}= \begin{cases}1, & \text { if } i_{s} \geq \text { threshold, } \\
0, & \text { otherwise. }\end{cases} \\
\text { SuitIrr }=\frac{\sum_{s=1}^{n} i_{s} \cdot A_{s} \cdot t_{s}}{\sum_{s=1}^{n} A_{s} \cdot t_{s}} & \text { with } t_{s}= \begin{cases}1, & \text { if } i_{s} \geq \text { threshold, } \\
0, & \text { otherwise. }\end{cases}
\end{array}
$$

\footnotetext{
${ }^{12}$ https://energyplus.net/weather-location/europe_wmo_region_6/CHE/CHE_Geneva. 067000_IWEC, last accessed on March 2017.
} 


\section{increase of their efficiency.}

On the basis of the previous equations, we calculated the metrics that we used to evaluate the uncertainty of a grid at spacing interval $g=x[\mathrm{~m}]$ with regards to the reference grid at a spacing interval of $g=0.5[\mathrm{~m}]$ :

$$
\begin{gathered}
\text { AreaRatio }=\frac{\text { SuitArea }_{g=x}}{\text { SuitArea }_{g=0.5}} \\
\text { IrrRatio }=\frac{\text { SuitIrr }}{\text { SuitIr }_{g=0.5}} \\
\text { IrrDiff }=\text { SuitIrr }_{g=x}-\text { SuitIrr }_{g=0.5}
\end{gathered}
$$

Similarly, we calculated also the Root Mean Square Error (RMSE) and Relative Root Mean Square Error (RRMSE) for each surface $s$ :

$$
\begin{aligned}
R M S E & =\sqrt{\frac{1}{n} \sum_{s=1}^{n}\left(\text { SuitIrr }_{s, g=x}-\text { SuitIrr }_{s, g=0.5}\right)^{2}} \\
R R M S E & =\frac{\sqrt{\frac{1}{n} \sum_{s=1}^{n}\left(\text { SuitIrr }_{s, g=x}-\text { SuitIr }_{s, g=0.5}\right)^{2}}}{\frac{1}{n} \sum_{s=1}^{n} \text { SuitIrr }_{s, g=x}}
\end{aligned}
$$

It should be noted that we considered in this analysis only surfaces that have at least one sensor at each grid spacing, in order to compare a consistent set of surfaces. The considered surfaces are rendered with gradient false colors in Figure 9.

\subsection{Comparative analysis against Radiance-based simulations}

Daysim is based on the daylight coefficient method determining an interpolation of the sun positions (Reinhart \& Walkenhorst, 2001). For the considered climate, only 65 sun positions are taken into consideration. Even if this is usually considered acceptable for annual calculations, it might affect the accuracy of the simulation, 
In order to test the accuracy of the simulations, we run a simulation using Radiance's gendaylit and rtrace on each daylit hour of the year. Since the computational cost for an annual simulation in Radiance is much greater than in Daysim, the validation in Radiance was limited to one sample building (ID 54 in Fig. 2b), which has some typical characteristics of this urban area: gable roof with a dormer, one façade facing a courtyard and another one facing the street, while other two façades are adjacent to the contiguous buildings.

We used the same geometry and material definitions used in the simulation in Daysim. Daysim and Radiance share in fact the same data format ( *.rad). However, for this comparative analysis, we included in the simulation only the sensor points belonging to the selected building.

We thus analyzed the results for the four surfaces of the building displayed in Fig. 5, so as to evaluate the accuracy of the simulations in Daysim compared to Radiance with regards to the specific purposes of this paper at both different spatial (whole building or single surfaces) and temporal annual and hourly time time-steps.
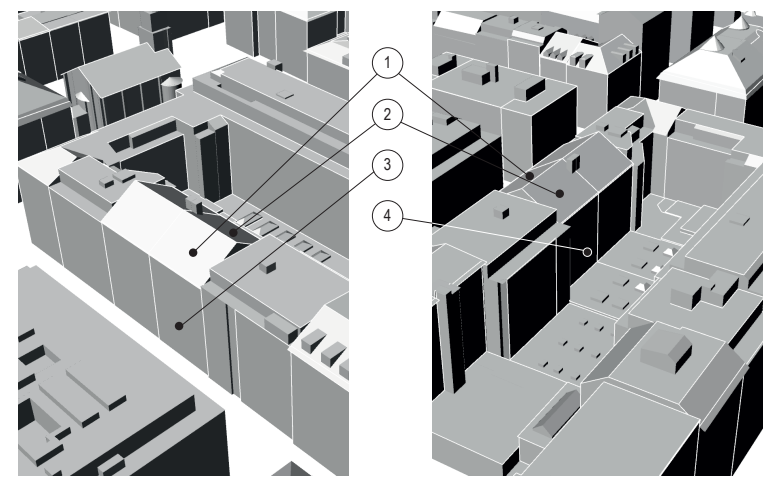

Figure 5: Legend for surfaces of the case-study building used in $\S 4$.

\section{Preliminary sensitivity analyses}

In this section, we present the results of preliminary sensitivity analyses to test the effectiveness of the methodology. The goal of this section is twofold: testing the sensitivity of simulations to some Radiance parameters used in the Daysim simulations and testing the results of Daysim against "pure" Radiance-based simulations (i.e. using gendaylit without daylight coefficients). As explained in $\S 3.6$, we conducted this test in a case-study building, i.e. a building of the analyzed tile which is considered to be representative of the urban conditions of the area and represented in Fig. 5. 


\subsection{Sensitivity to ambient bounces and accuracy}

We tested simulations in Daysim at increasing grid spacing using different Radiance parameters:

- ambient accuracy - aa

- ambient bounces $-a b$

The parameters values are listed in Table 3.

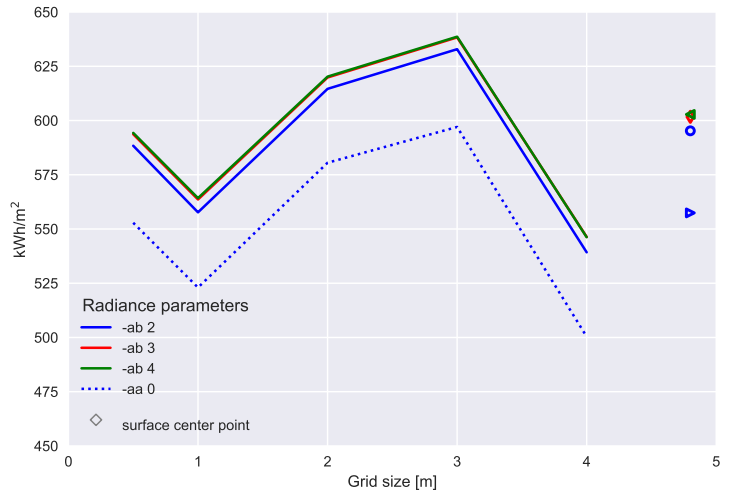

(a)

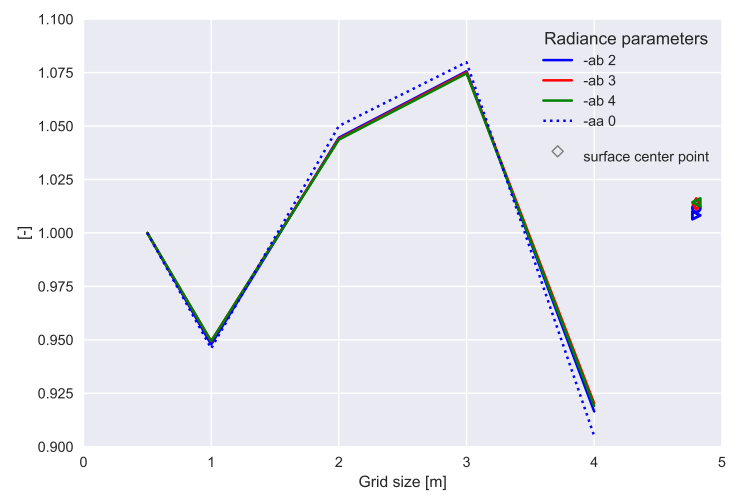

(b)

Figure 6: Suitable solar irradiation (a, see Eq. 2) and ratio of suitable solar irradiation (b, see Eq. 4) with spacing $g=x[\mathrm{~m}]$ to suitable solar irradiation with $g=0.5[\mathrm{~m}]$ for threshold $t=0$ $\left[\mathrm{kWh} / \mathrm{m}^{2}\right]$ (b). Please note that, for better visualization, the results for sensor points placed at the surfaces' centers (unfilled markers) have been arbitrarily plotted with $x=4.80 \mathrm{~m}$. As expected, the higher the ambient bounces, the higher the irradiation; conversely, with an ambient accuracy set to 0 , the irradiation is significantly lower than with the default parameter of 0.1 . However, for both parameters, we notice similar trends regarding the effect of the grid spacing. 
Table 3: Tested Radiance parameters (the other parameters are the same as in Tab. 2), and grid size. Ambient bounces - $a b$ parameter varies from 2 (i.e., 1 bounce onto the $3 \mathrm{D}$ scene before reaching the sky vault) to 4 (i.e., 3 bounces). Ambient accuracy -aa parameter varies from 0.1 (i.e., low error interpolation) to 0 (i.e., no interpolation).

$\begin{array}{lll}-a b & -a a & \text { Grid size }[\mathrm{m}] \\ 2 & 0.1 & 0.5 \\ 2 & 0.1 & 1 \\ 2 & 0.1 & 2 \\ 2 & 0.1 & 3 \\ 2 & 0.1 & 4 \\ 2 & 0.1 & \text { surface center } \\ 3 & 0.1 & 0.5 \\ 3 & 0.1 & 1 \\ 3 & 0.1 & 2 \\ 3 & 0.1 & 3 \\ 3 & 0.1 & 4 \\ 3 & 0.1 & \text { surface center } \\ 4 & 0.1 & 0.5 \\ 4 & 0.1 & 1 \\ 4 & 0.1 & 2 \\ 4 & 0.1 & 3 \\ 4 & 0.1 & 4 \\ 4 & 0.1 & \text { surface center } \\ 2 & 0 & 0.5 \\ 2 & 0 & 1 \\ 2 & 0 & 2 \\ 2 & 0 & 3 \\ 2 & 0 & 4 \\ 2 & 0 & \text { surface center }\end{array}$

As can be seen in Fig. 6a, the choice of the parameter influences the received solar irradiation. By setting - $a a$ to 0 , we notice a reduction of about $10 \%$. However, this reduction is consistent at all spacing intervals. Similarly, we notice an increase of annual solar irradiation by using higher $-a b$ parameters than the default one of $-a b 2$. This is expected because the augmentation of number of bounces augments the quantity of reflected solar radiation that is taken into account. However, the increase is not significant and, as for $-a a$, is consistent at all spacing intervals.

\subsection{Sensitivity to grid spacing}

The ratios of irradiance values calculated in Radiance and Daysim are first presented for each hour of the year. In Fig. 7, we can see that the frequency increases along with the increasing grid spacing. This means that the accuracy of Daysim simulations is decreasing with increasing grid spacing. Because of the discrete sun 
positions, a sparser sensor points distribution is more likely prone to errors due to the discrete sun positions (65), while for lower spacing intervals each surface is represented by more sensor nodes, the error being then more easily compensated. In Fig. 7 we can also see that the South-facing façade (surface 3) is the most affected by the simplifications of Daysim, and this is especially visible in winter where we can see a frequent overestimation of hourly values, especially starting from a grid spacing equal to $3 \mathrm{~m}$. Conversely, on the North-façade oriented towards the courtyard (surface 4) the irradiation is generally - constantly in the case of a grid spacing equal to 3 or 4 m - overestimated.

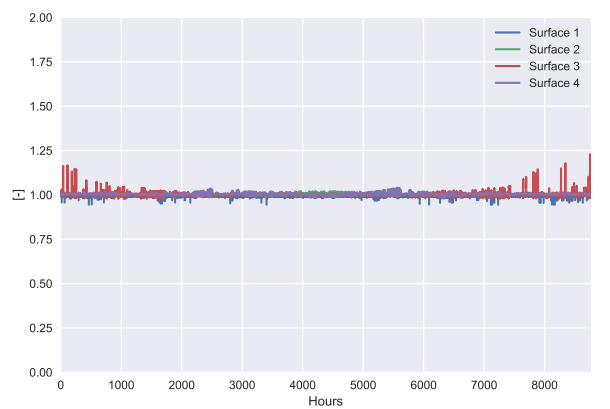

(a)

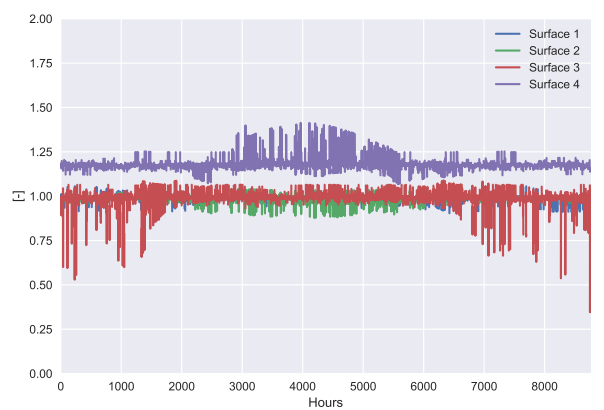

(c)

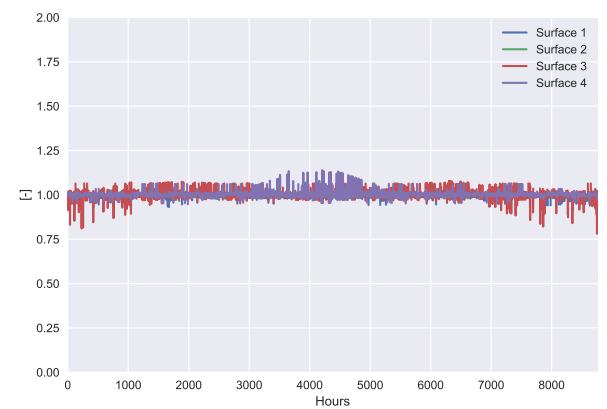

(b)

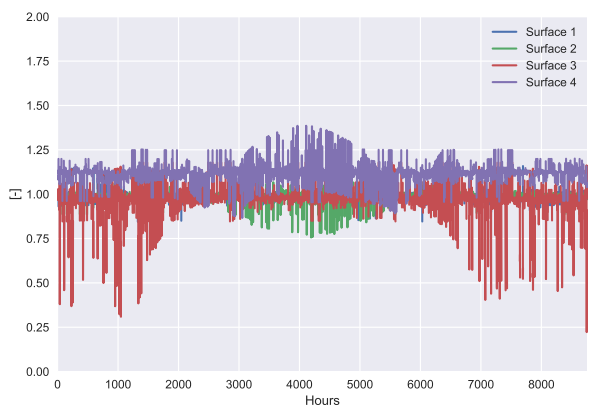

(d)

Figure 7: Ratio of hourly solar irradiance values calculated in Radiance to those calculated in Daysim at different grid sizes: a) $=1 \mathrm{~m}, \mathrm{~b})=2 \mathrm{~m}, \mathrm{c})=3 \mathrm{~m}, \mathrm{~d})=4 \mathrm{~m}$ ). See Fig. 5 for reference to surface numbers. Starting at a spacing interval of $3 \mathrm{~m}$, we can notice significant discrepancies between results in Daysim and Radiance.

The annual results presented in Fig. 8 show that trends for Daysim and Radiance results are similar and the curves mostly coincident. However, starting from a grid spacing equal to $4 \mathrm{~m}$, there is an underestimation in Daysim results compared to Radiance ones. This is probably caused by irradiation on the South-façade being 
most of the time underestimated.

We also notice that the error calculated by both simulation engines is negligible till a $2 \mathrm{~m}$ spacing interval, as the two curves are mostly coincident. Similarly, in the graphs of hourly results, the range of variation for grid at $1 \mathrm{~m}$ and at $2 \mathrm{~m}$ is very similar and always contained between 0.5 and 1.5 , while being significantly wider for coarser grids.

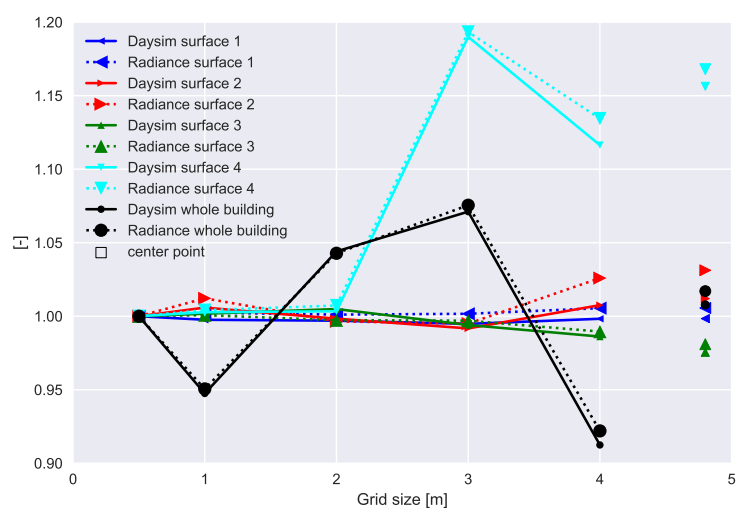

Figure 8: Ratio of suitable solar irradiation (see Eq. 4) with spacing $g=x[\mathrm{~m}]$ to suitable solar irradiation with $g=0.5[\mathrm{~m}]$ with threshold $t=0\left[\mathrm{kWh} / \mathrm{m}^{2}\right]$ for different simulations run with Daysim and Radiance on a structured grid. See Fig. 5 for reference to surface numbers. Please note that, for better visualization, results for sensor points placed at the surfaces' centers (unfilled markers) have been arbitrarily plotted with $x=4.8[\mathrm{~m}]$. We can see that the trend is similar for simulations run with Radiance and Daysim.

\section{Results}

We present here the results of the analysis at different spatial resolutions, starting from annual results calculated on the entire urban sector down to a more detailed analysis per surface, through both comprehensive plots and spatial maps. Fig. 9 shows a perspective view of the analyzed 3D model of the urban area with some error metrics visualized as false-colors on the building surfaces. 


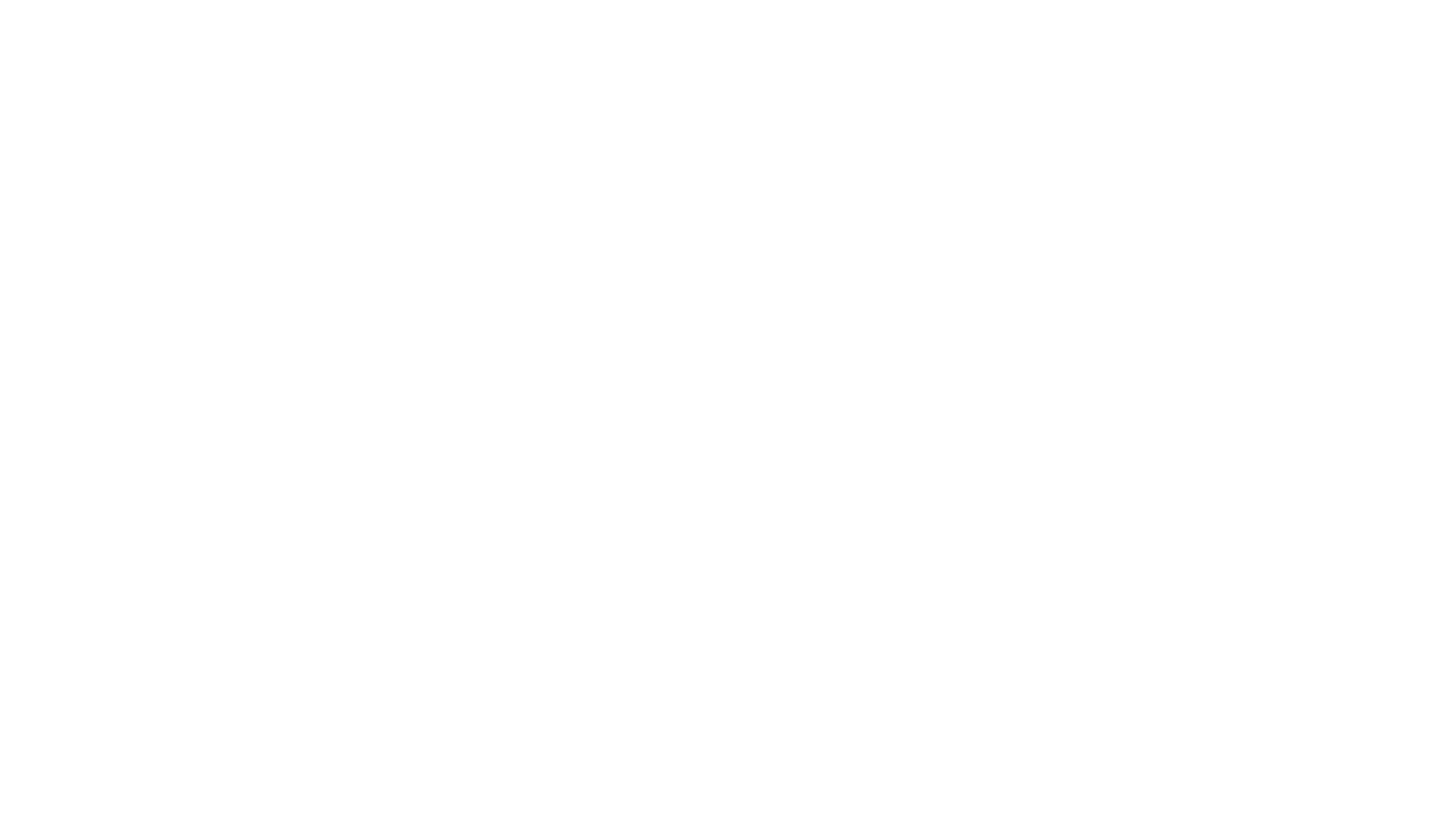

(a)

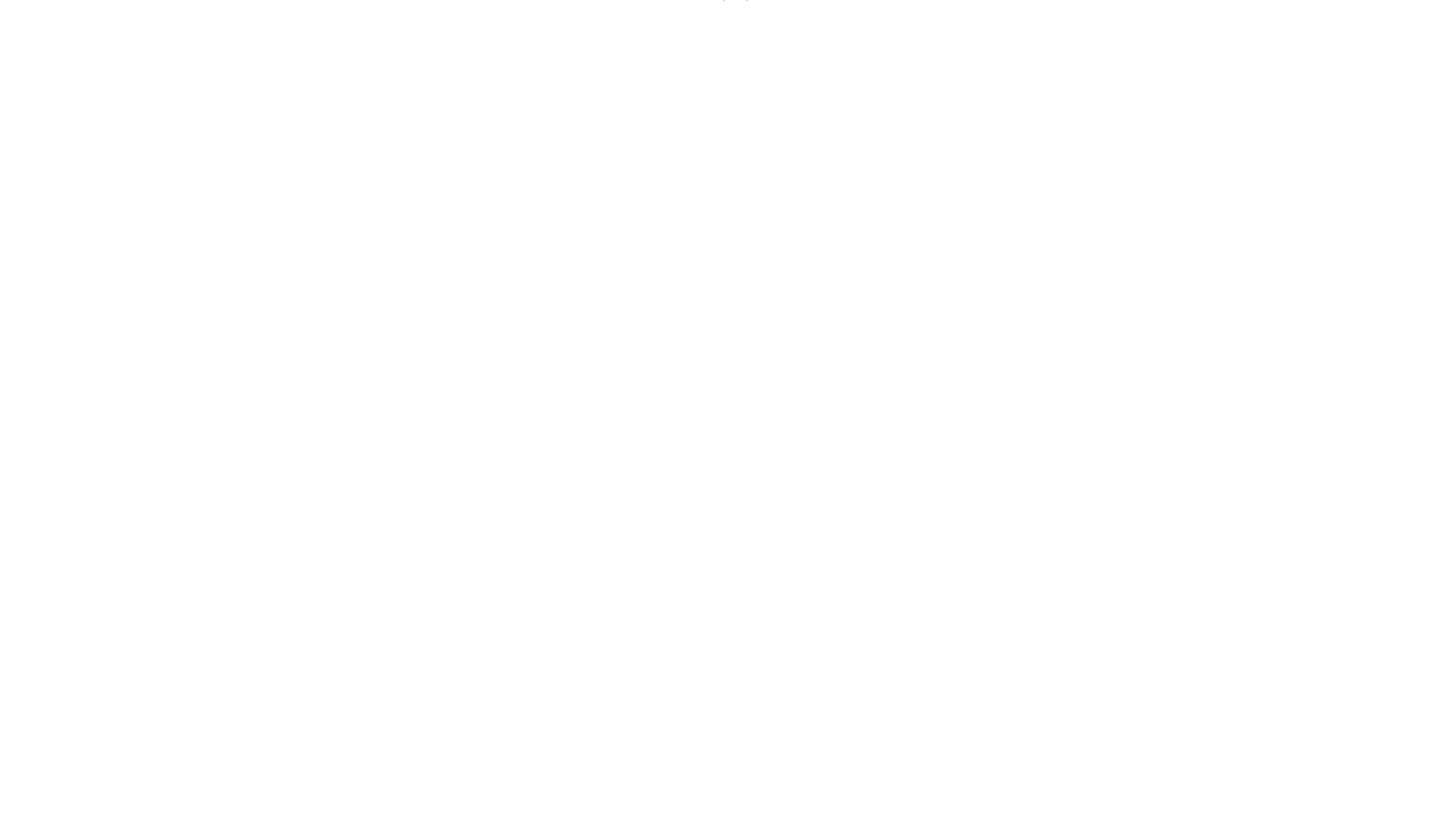

(b)

Figure 9: Ratio of suitable solar irradiation (a, see Eq. 4) and difference of suitable solar irradiation (b, see Eq. 5) at resolution $g=x[\mathrm{~m}]$ (indicated in the bottom-right corner) and at reference resolution $g=0.5[\mathrm{~m}]$ with threshold $t=0\left[\mathrm{kWh} / \mathrm{m}^{2}\right]$ on a structured grid. An animation presenting the results for the different resolutions can be s2en using Adobe Acrobat or Adobe Reader. The false-color maps are based on data extracted from the Système d'information du territoire à Genève (SITG), as of June 2016. 


\subsection{Urban-scale analysis}

As can be seen in Figs. 10 and 11, the use of a structured or unstructured grid has a huge impact on the results. This is because the unstructured grid keeps the area almost stable at all spacing intervals, as mesh faces cover the whole building envelope, while the panelization process determines a decrease of the available area (Fig. 3b). Consequently, this causes also a significant decrease in the number of sensor points, which does not occur in the case of an unstructured grid (Fig. 3a).

By normalizing the results of the structured grid by the area available at $t=0$ $\left[\mathrm{kWh} / \mathrm{m}^{2}\right]$, a decrease for most of the thresholds can be noticed, but with a much smaller magnitude (Fig. 10c). We can assume such normalized results are more realistic, as they consider only the actual panel surfaces. It can be also noticed that the surface decreases more steeply for high thresholds, because of the losses of highly irradiated surfaces that are available at $t=0\left[\mathrm{kWh} / \mathrm{m}^{2}\right]$.

However, with respect to the suitable irradiation (Fig. 11b) lower thresholds present more losses. For both area and irradiation, decreasing curves along with the increasing grid size can be observed. This means that we tend to underestimate the available irradiation and the suitable are, if the $0.5-\mathrm{m}$ grid is considered as the reference. However, this is not always the case if the analysis is limited only to low thresholds (in terms of area) and high thresholds (in terms of irradiation). In this case, the variation is negligible and in some cases even positive. Moreover, the surface reduction caused by coarser resolutions is more significant for high thresholds, while, on the contrary, in terms of irradiation low thresholds are more affected. 


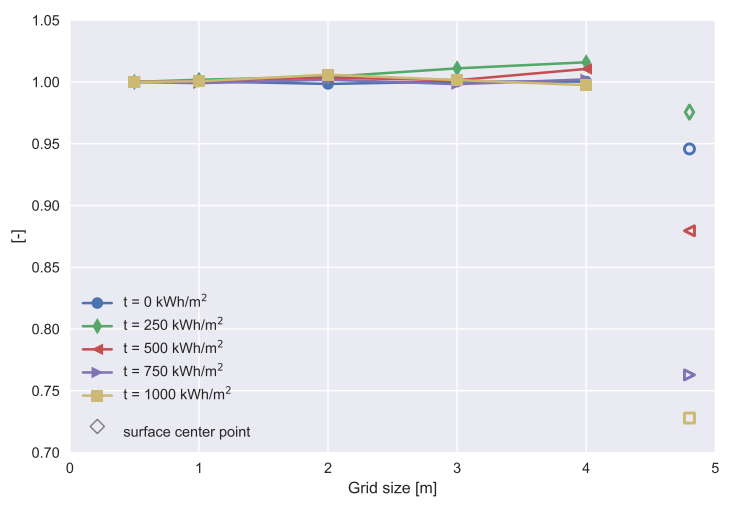

(a)

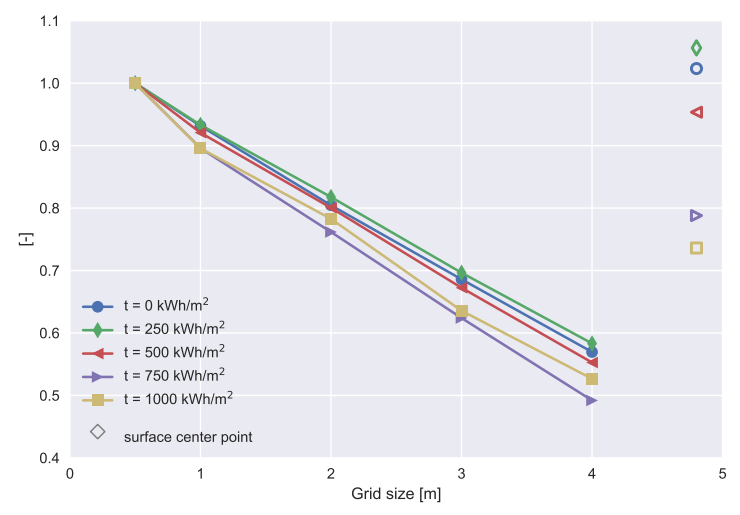

(b)

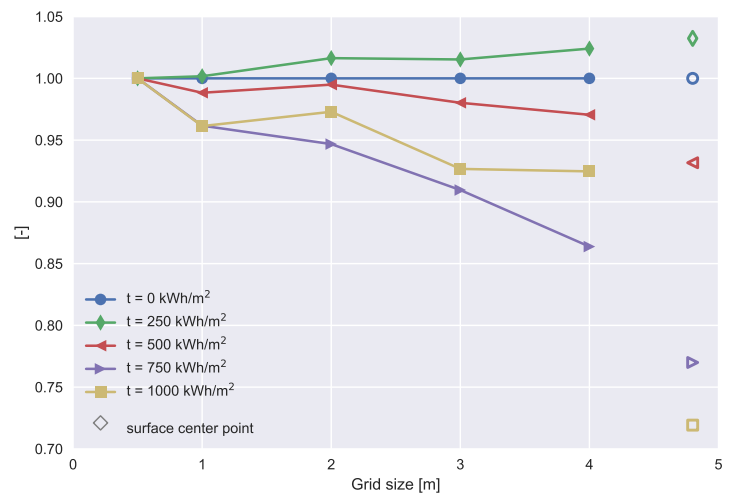

(c)

Figure 10: Ratio of suitable surface (see Eq. 3) at spacing $g=x[\mathrm{~m}]$ to suitable surface with resolution $g=0.5[\mathrm{~m}]$ for different thresholds $t\left[\mathrm{kWh} / \mathrm{m}^{2}\right]$. Subfigure a) shows results for an unstructured grid, b) for a structured grid and c) for a structured grid normalized by $t=0$ $\left[\mathrm{kWh} / \mathrm{m}^{2}\right]$. Please note that, for better visualization, the results for sensor points placed at the surfaces' centers (unfilled markers) have been arbitrarily plotted with $x=4.8[\mathrm{~m}]$ and that the $\mathrm{y}$-scale is not constant across the three graphs. In the case of the structured grid (b), the suitable surface is inversely correlated with the grid spacing (cf. Fig 3b). 


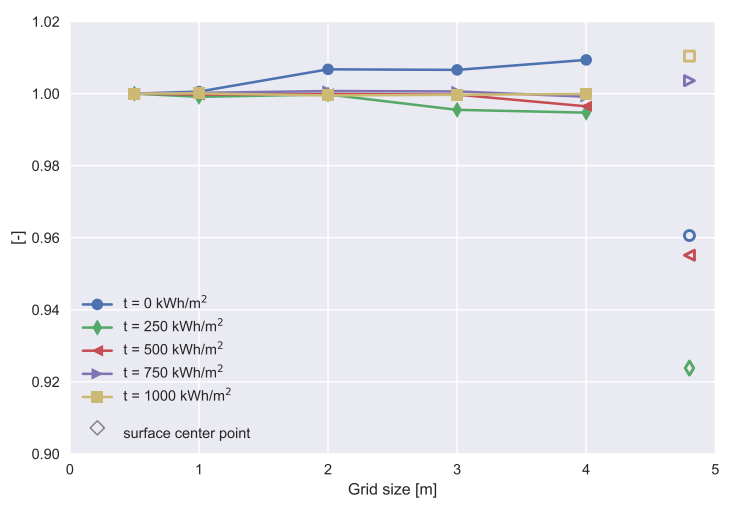

(a)

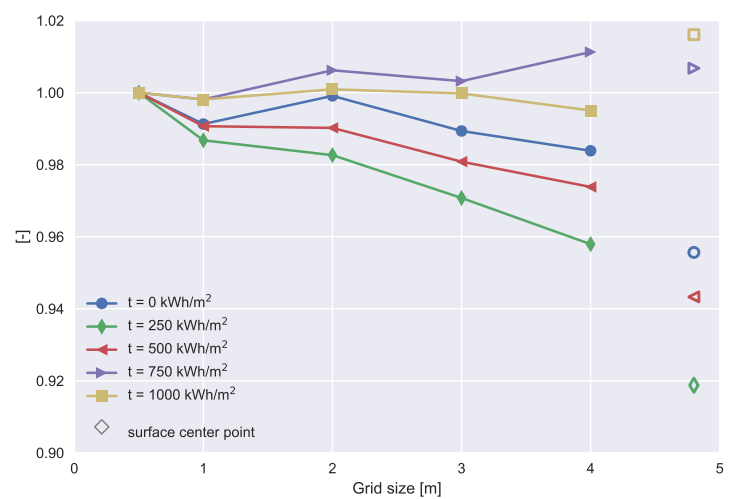

(b)

Figure 11: Ratio of solar irradiation (see Eq. 4) with resolution $g=x[\mathrm{~m}]$ to annual solar irradiation with $g=0.5[\mathrm{~m}]$ for different thresholds $t\left[\mathrm{kWh} / \mathrm{m}^{2}\right]$. Subfigure a) shows results for an unstructured grid, b) for a structured grid. The results for sensor points placed at the surfaces' centers (unfilled markers) have been arbitrarily plotted with $x=4.8[\mathrm{~m}]$.

\subsection{Comprehensive surface analysis}

If we analyze the difference in irradiation for each surface IrrDiff (see Eq. 5 with threshold $t=0\left[\mathrm{kWh} / \mathrm{m}^{2}\right]$ for a structured grid) plotted in Fig. 12, we can notice an increase of the median and upper quartile. This means that the annual irradiation per surface is more likely to be overestimated with regards to the $0.5-\mathrm{m}$ grid. By looking at the distribution of the variation in suitable solar irradiation (Fig. 14), we can also see that the negative solution space gets bigger while increasing the grid size (from subfigures a to e). This could explain why the cumulative results show a decreasing trend, while the results per surface have an increasing trend: by increasing the grid spacing, surfaces with a strong negative variation occur more frequently, while there is an almost stable median positive variation. 


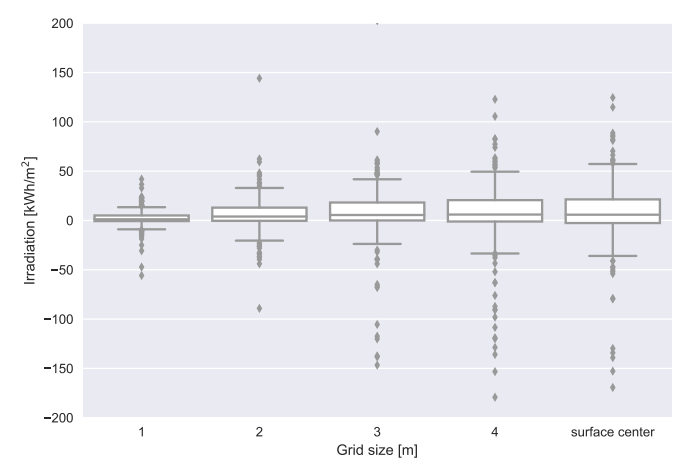

Figure 12: Difference of suitable annual solar irradiation (see Eq. 5) with threshold $t=0\left[\mathrm{kWh} / \mathrm{m}^{2}\right]$ for a structured grid. Please note that some data points are outside the y-limits. Surface irradiation is generally overestimated using coarser grids, while there is an increasing number of surfaces whose irradiation is highly-underestimated. The median difference is stable starting from a 3-m resolution.

If we consider the RRMSE (Figs. 13c and 13d) calculated on each surface, we find higher error values that the one seen in the previous section for the ratio of available solar irradiation. The maximum RRMSE is still low, though: $7 \%$ for $g=400$ and $t=0$, corresponding to a RMSE of $45 \mathrm{kWh} / \mathrm{m}^{2}$, for a structured grid. Unlike for the ratio of available solar irradiation, for a structured grid we can see that the error increases at all thresholds with a linear trend with decrease of the resolution, while for an unstructured grid it is stable for all resolutions lower than $1 \mathrm{~m}$. The trend of the structured grid could be due to specificities of the algorithm already highlighted in §3.3: with increase of spacing, the offset from the border of the surfaces also increases, causing losses in the upper part of the façade. 


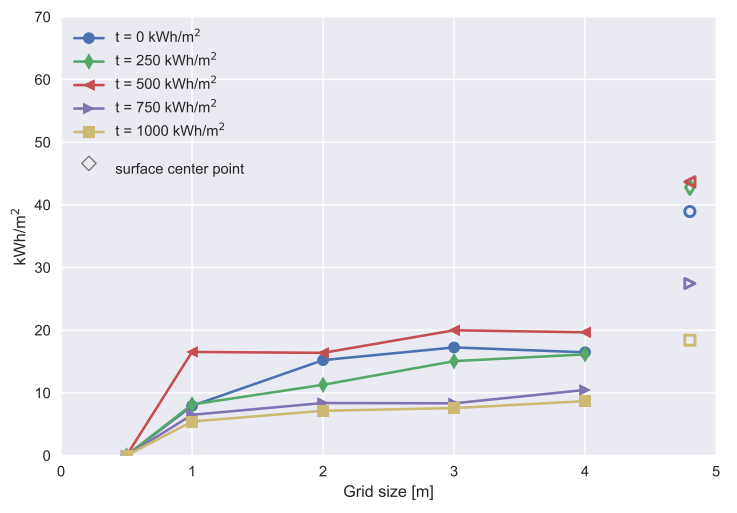

(a)

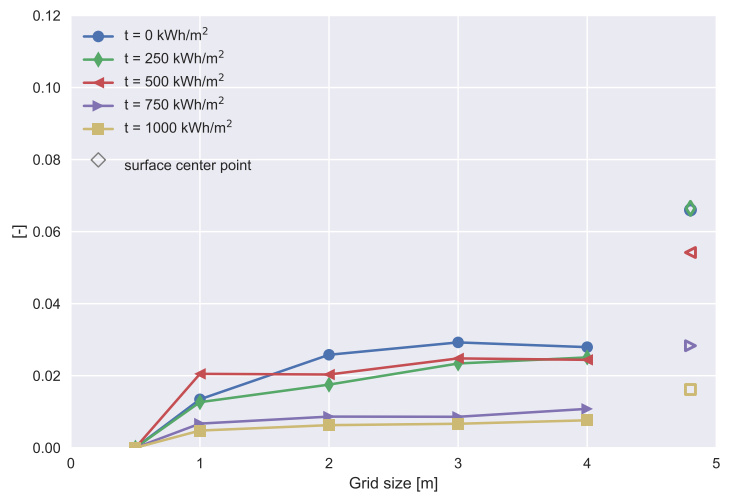

(c)

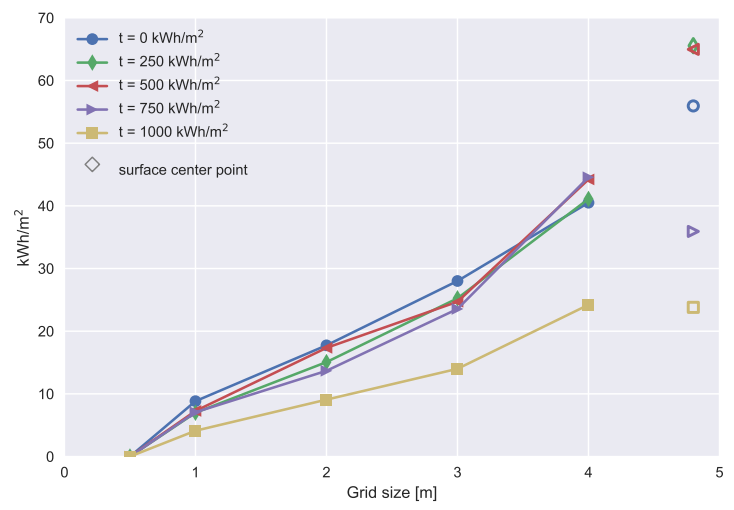

(b)

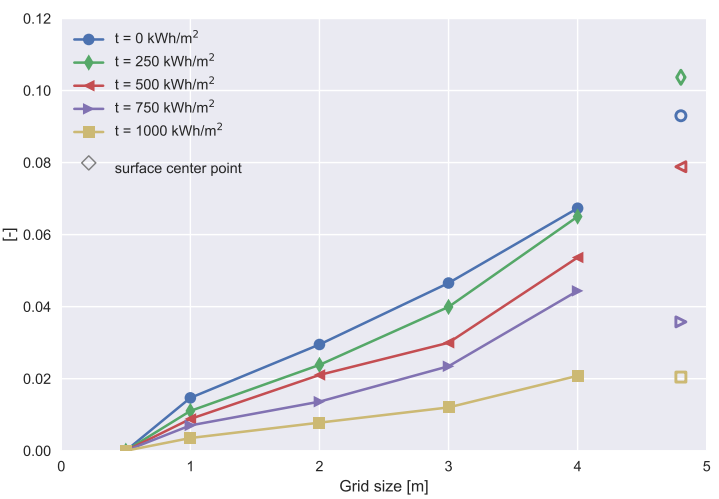

(d)

Figure 13: In the first row, Root Mean Square Error (see Eq. 6), and, in the second row, Relative Root Mean Square Error (see Eq. 7) for resolution $g=x[\mathrm{~m}]$ and reference values calculated at $g=0.5[\mathrm{~m}]$, for different thresholds $t$. Subfigures a) and c) show results for an unstructured grid, b) and d) for a structured grid. The results for sensor points placed at the surfaces' centers (unfilled markers) have been arbitrarily plotted with $x=4.8[\mathrm{~m}]$. Results for the unstructured grid present a mostly-stable error, while for the structured grid it is increasing with the grid spacing.

\subsection{Spatial surface analysis}

In Fig. 9b we clearly see some roof surfaces with constructions presenting a strong decrease in irradiation. This happens for example when in low-definition grids the sensor points are shaded by the construction, while in the reference grid other wellexposed sensor points counter balanced the results. Those surfaces present a strong decrease, while overall most of the surfaces slightly increase their irradiation, in particular those South-facing and the façades. This seems to confirm the results that we have seen in Fig. 12. 
By looking at Fig. 14, we can see that the difference in solar irradiation is generally greater on façades than on rooftops. Similarly, North-facing façades present a smaller variation compared to the other orientations. This corresponds to the expectations, as vertical and North-facing surfaces have a less favorable solar exposition than roofs. If we exclude North-facing surfaces, we cannot see any significant variation due the orientation. Nevertheless, the points corresponding to flat roofs have the greatest variation (both negative and positive) at all resolutions.

We can see in Fig. 14b, that the distribution of range of values is very similar to that of Fig. 14a, and always under $100 \mathrm{~kW} / \mathrm{m}^{2}$, except for one data point, which is located on a vertical surface. Starting from $14 \mathrm{c}$ the difference in values become more significant and in many cases this is higher than $100 \mathrm{~kW} / \mathrm{m}^{2}$.

As already seen in Fig. 12, most of the surfaces present an increase of solar irradiation. However, the extremes can be observed in the lower part of the plots of Fig. 14, in particular with low-resolution grids. Again, these results explain why we observe a general decrease in solar radiation at the urban level - i.e. a limited number of surfaces with huge losses -, and an increase at the surface level - i.e. the majority of surfaces presenting a positive variation. 


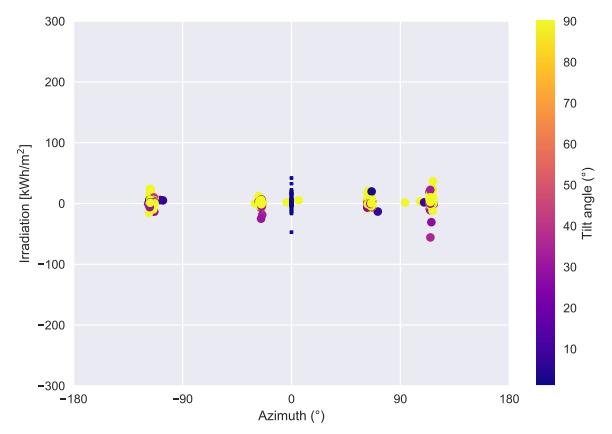

(a)

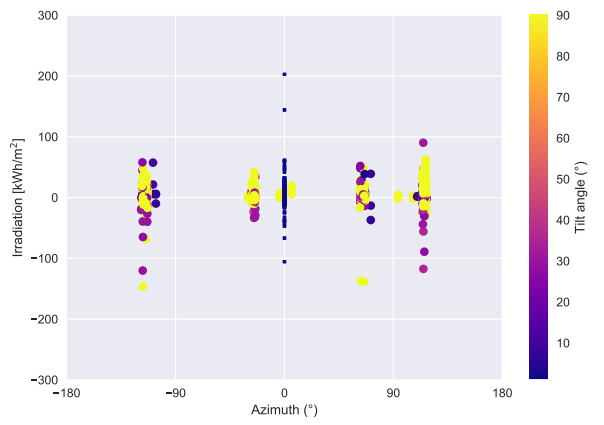

(c)

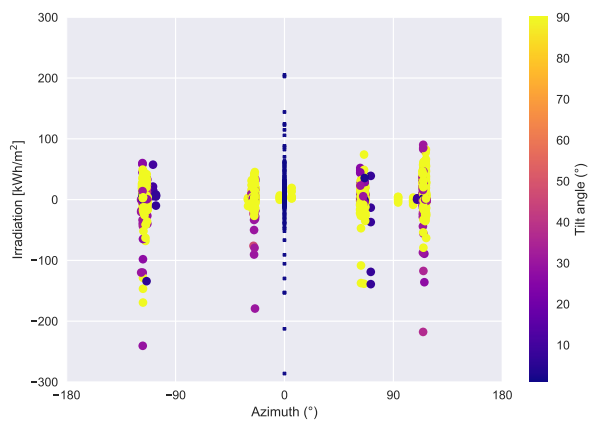

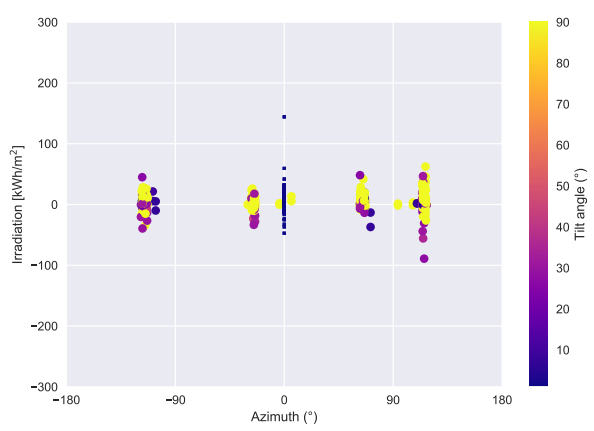

(b)

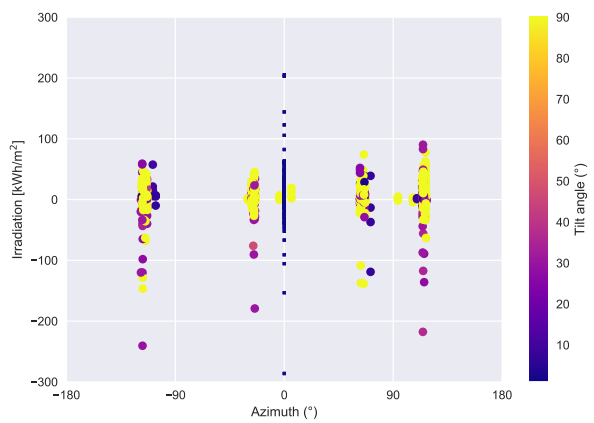

(d)

(e)

Figure 14: Difference of suitable solar irradiation (see Eq. 5), depending on orientation (x-axis) and tilt angle (colors), with threshold $t=0$, for a structured grid at increasing spacing: a) $1 \mathrm{~m}, \mathrm{~b}$ ) $2 \mathrm{~m}$, c) $3 \mathrm{~m}$, d) $4 \mathrm{~m}$, e) surface center. Some data points in Fig. 14e are outside the y-limits. The squared data points represent horizontal surfaces (i.e. they do not have an azimuth) and have been arbitrarily set to $x=0^{\circ}$. Horizontal surfaces present the highest error values (both positive and negative). Among tilted surfaces, South-facing surfaces present as expected higher absolute error values. With increasing grid spacing, we can notice an increasing number of highly-underestimated irradiation at all orientation and tilts. 


\section{Discussion}

The scope of this work was the investigation of the impact of 3D model discretization on the calculated solar irradiation. In the previous sections, we have analyzed the results using different metrics and analysis targets. In the light of these results, we will discuss here the significance of these findings for solar assessments in urban environments and compare them to prior works. We will finally list the shortcomings related to the methodology and the improvements and extensions to be planned in future work.

\subsection{Significance of findings}

We have seen that the results vary across different thresholds and orientation (azimuth and tilt angle). This means that the significance of the findings for solar energy assessments depends on the specific conditions in which solar modules are installed.

Thresholds represent a typical assessment method for evaluating the PV-suitability of surfaces (Kanters et al., 2014). A minimum annual irradiation is needed in fact for the economic viability of a (BI)PV installation. However, the viability thresholds are expected to lower in the upcoming years, due to lower prices of photovoltaics as well as of increasing efficiency. We can hence consider that lower thresholds represent a benchmark for future assessments. In this sense, the results show that the uncertainty of the actual solar yield of building surfaces will have an increasing importance in the future, as the difference in the results increases when considering lower thresholds. Conversely, for typical urban-scale assessments at present conditions only considering surfaces with more than $1000 \mathrm{kWh} / \mathrm{m}^{2}$ per year, a low resolution is sufficient for having a good accuracy.

The orientation is related to the threshold. Vertical and North-exposed surfaces do not attain higher thresholds. In terms of vertical angle, facades have a higher relative variation with regards to the $0.5 \mathrm{~m}$ resolution grid. For this reason, we can assume that they also need smaller grid spacing in order to achieve good accuracy. However, a smaller grid spacing on façades is probably overkill unless a higher LOD (including for example windows and balconies) is used.

In terms of tilt angle, roofs present greater difference with regards to the reference grid because they also have higher irradiation values. At all resolutions, flat roofs present significant variations, probably because of the presence of many roof superconstructions, whose shading effect is correctly represented only at very high resolutions.

In general, the error values found in this work are relatively small (e.g. 0.07 RRMSE for a 4-m spacing). We acknowledge that other uncertainty factors, such 
as, for example, the Level of Detail and reflective properties of surfaces, might have a higher impact than the ones analyzed in this work. However, we should consider that the grid size (as well as the ambient parameters) are freely set by the user and coarser settings are only determined by the need of reducing the computational cost. Differently, some other parameters rely on the quality of information that is available at the urban scale. In this sense, the use of standard reflective values is normally acknowledged in the literature (e.g. in Jakubiec \& Reinhart (2013); Fath et al. (2015)), as this information is hardly obtainable for each single building.

\subsection{Comparison to relevant prior art}

Similarly to what observed by Peronato et al. (2016b) regarding the effect of vegetation on solar irradiation, also in this study we noticed that results are highly influenced by the analysis granularity. At the scale of the analyzed urban level, the difference between different grid resolutions is mostly negligible, while at the surface level we noticed a large variation in the results, which intuitively increases with the coarser grids. This is because, at the large scale, underestimations partially compensate overestimations of solar irradiation. On the contrary, at the surface level, the behavior of the grid at the different scales is unpredictable, as depends, among other factors, on the shape of the surface and the presence of obstructions.

As Alam et al. (2016), we also noticed that shaded areas are those more impacted by the effect of resolution, at least in relative terms. As in their work, we saw that solar irradiation per building is not constantly over-estimated or under-estimated, but it depends on the specific building situation.

As both Alam et al. (2016) and Bremer et al. (2016) already noticed, we also confirmed that the accuracy of results decreases when using coarser grids. However, in terms of calculated relative errors, we have found smaller values than those obtained by Bremer et al. (2016) (in their case, 10\% of mean solar irradiation for a 1-m sensor grid resolution). This difference might be due to the different characteristics 5 of the urban areas and calculation methods (e.g. in their case the obstructions are defined as voxels and the model does not account for reflected radiation).

\subsection{Limitations}

The possibility of generalizing the results to other urban areas is hard to assess. We can assume that parameters that might influence the results are built density,

building typology (including roof type), street aspect ratio and the presence of roof top constructions. The characteristics of the analyzed area are common in many historical cities in Europe and we can thus expect similar results. Moreover, due to the specific characteristics, such as high built density and presence of roof-top constructions, we can expect that the findings of this study could represent a worst-case 
scenario. Similarly, the calculated irradiation ratio and RRMSE should be directly comparable to other locations with similar characteristics. However, the absolute difference and the RMSE calculated in this paper is clearly climate-dependent, therefore we cannot extrapolate this information to other contexts, unless it is normalized by the local mean irradiance.

Concerning the limitations of the model, the 3D model we used provides a quite accurate representation of reality, as it includes the actual roof shape as well as rooftop superstructures, but, as in LOD2 specifications, windows and balconies are not modeled. For this reason the area which is here considered as available for installing solar PV is probably overestimated. However, since we analyzed the surfacenormalized values and considering that windows are usually homogeneously distributed on the façades, we can assume that the results are not significantly affected.

With regards to the method for testing the sensitivity of the results, we here used a simple parametric analysis focusing on the resolution of the sensor grid and its arrangement. Therefore, we generally used standard values for the other simulationspecific parameters, such as, for instance, the reflectivity of the materials and the number of inter-reflections. However, these and other parameters might have a combined effect with the grid resolution and arrangement, which was not investigated in this work.

\subsection{Outlook}

This work provided some hints about the importance of spatial position (e.g. tilt and azimuth) of the surfaces in the definition of the correct resolution of the sensor grid. In this sense, future work should investigate varying the resolution considering the actual need for higher resolutions (i.e. smaller spacing), similarly to the concept of unstructured adaptive methods applied in CFD analysis to maximize accuracy and efficiency (Chung, 2002). In addition, to the spatial analysis that can be conducted by analyzing the normal vectors of the sensor points, other simulation-based results could be used as proxies to evaluate the need for higher resolutions. For example, the sky view factor (SVF) is relatively fast to calculate even at grids with small spacing since it is time- and weather-independent. It can hence provide an assessment of the complexity of the shading conditions of a surface, determining whether the high resolution is actually needed also for more computationally-expensive simulations, such as those of hourly irradiance values. Similarly, low-resolution simulations (using for example a cumulative sky or coarser ambient bounces or ambient accuracy settings) can also be used to define the needs for higher resolutions.

We have seen that the highest variations in our results are usually caused by super-constructions on rooftops. Since the presence of these depend on the level 
of detail (LOD) of the geometric model, we can assume that the resolution of the grid is influenced by the LOD. In case a higher LOD is used, a higher resolution of the grid should also be implemented. Conversely, a coarser grid should be sufficient when analyzing 3D models at lower LOD. Prospective work should investigate this hypothesis in order to quantify the optimal sensor grid resolution for each LOD. At this stage, we assumed that a high-resolution grid would be still beneficial to analyze a BIPV potential on façades, considering that these can be installed also on windows and balconies.

With respect to the LOD of the model used in this work and the other characteristics of the analyzed case study, this work provided a quantification of the error that has to be considered when using coarser sensor grids. However, this is only one of the factors affecting the accuracy of the predicted solar irradiation, as shown in $\S 2.1$. Future work should provide a comprehensive sensitivity analysis on simulation parameters so as to be able to rank them by effect magnitude or combine the error they induce in an overall confidence interval.

The work used a simulated ground truth the to assess the effect of the grid size. This method allowed a large-scale application on an urban tile, which would be unfeasible using physical measurements. However, this type of analysis can be complemented with a measurement campaign on a smaller area to validate the findings.

The information about uncertainty is now unavailable in decision-making tools such as solar cadasters, while it can be crucial when comparing multiple planning scenarios. For this reason, we plan to include the information about the uncertainty due to grid resolution in the evaluation of solar energy planning scenarios in order to check its significance in the decision-making process.

\section{Conclusion}

This paper analyzed the impact of the sensor grid resolution and its spatial arrangement on solar irradiation. The scope of this work was twofold:

1. defining the grid resolution allowing the best trade-off between accuracy and the number of sensor points (i.e. a proxy of computational cost) for urban-scale assessments

2. assessing the error that has to be considered, with regards to reference parameters, while using coarser resolutions.

We highlighted the importance of the grid arrangement and - to some extent 675

- its resolution in the calculated solar irradiation. Results show that a structured grid is much more affected by resolution, as the number of sensor points substantially 
decreases along with the space interval. However, the decrease of sensor points potentially determines also a saving in the simulation time and allows an early evaluation of PV-suitable surfaces by excluding those that are too small.

We have also seen the importance of the scale at which results are analyzed. Sensitivity to the resolution augments with analysis at higher granularity. However, the median irradiation difference with the reference grid is not significantly varying between the thresholds.

The results of the comparison with Radiance show that the method proposed in this paper is more robust when using small spacing intervals, as it is less subjected to errors due to the simulation engine. The results of the validation showed also the error up to a 2-m spacing interval is negligible, even at higher spatial or temporal granularities. In this sense, we can conclude that the results showed in this paper are also accurate till a $2-\mathrm{m}$ resolution, while they could be biased due to simplifications in the simulation tool at coarser resolutions. We also noticed that the results are more affected by the simplifications of the simulation engine when analyzing hourly values (especially in winter) and surfaces, especially the inner and North-facing (azimuth $\sim 25^{\circ}$ ) ones.

On the basis of the results and considering the limitations discussed in the previous section, we can finally provide some answers to the research questions listed in the introduction.

At which resolution does the error become acceptable? All considered resolutions (0.5 to $4 \mathrm{~m})$ provided acceptable deviations from the reference results $(0.5 \mathrm{~m})$. However, for resolutions higher than $2 \mathrm{~m}$, the error due to the simulation engine significantly affect the results and should be hence avoided. The choice can also be motivated by the fact the size of the long-side of a PV modules is generally smaller than $2 \mathrm{~m}$ (the median value from the CEC database (CEC, 2018) is $1.65 \mathrm{~m}$ ).

Are solar irradiation and PV-suitable area over- or under-estimated when using lowresolution grids? When analyzing the results for the entire urban area, irradiation is slightly underestimated using coarser grids. However, if the results are analyzed per surface, most of them have overestimated results in terms of solar irradiation. This is likely caused by the significant losses due to shading of roof-top constructions, which determine a underestimation of some specific surfaces affecting also the entire urban area.

${ }_{710}$ In which spatial and temporal conditions does the influence of grid resolution affect the results the most? Results are mostly affected during winter, which represent though a marginal part of the yearly solar irradiation. In relative terms, façades are 
more affected by resolution, while in absolute terms roofs show a stronger effect. In particular, roofs with over-constructions are in some cases highly affected by using a coarser grid $\left(<-100 \mathrm{kWh} / \mathrm{m}^{2} \cdot\right.$ year$)$.

Even accounting for the specificities of the analyzed urban area (in terms for example of morphology, architectural details and built density), we argue that the results of this study can be used to suggest an indicative spacing interval that provides a good trade-off between computational cost and accuracy of the results. Moreover, the same methodology can be also applied to a new location to check whether the proposed spacing interval is also optimal in other conditions.

We finally discussed that the information about the uncertainty due to grid resolution should be included in the decision-making process of solar energy assessments as a confidence interval. This would help urban and energy planners comparing different scenarios accounting for the uncertainty behind the results.

\section{Acknowledgments}

This work has been conducted in the framework of the ACTIVE INTERFACES research project (Rey et al., 2015), which is part of the National Research Programme "Energy Turnaround" (NRP 70) of the Swiss National Science Foundation (SNSF). Further information on the National Research Programme can be found at http: //www.nrp70.ch. G.P. acknowledges additional financial support from the Ecole polytechnique fédérale de Lausanne (EPFL).

We would like to thank Jan Wienold for providing us with the script for running annual simulations in Radiance, and Emilie Nault and Pietro Florio for their feedback on earlier versions of this manuscript.

We also thank Geneva's cantonal GIS office for releasing the geodata used for this work with an open license (Système d'information du territoire à Genève (SITG), extracted in June 2016).

\section{References}

Aguacil Moreno, S., Lufkin, S., \& Rey, E. (2016). Architectural design scenarios with building-integrated photovoltaic solutions in renovation processes: Case study in Neuchâtel (Switzerland). Proceedings of PLEA 2016, 32th international Conference on Passive and Low Energy Architecture, . URL: https://infoscience. epfl.ch/record/218887?ln=en. 
Alam, N., Coors, V., Zlatanova, S., \& van Oosterom, P. (2016). Resolution in photovoltaic potential computation. In ISPRS Annals of Photogrammetry, Remote Sensing and Spatial Information Sciences (pp. 89-96). Split, Croatia: Copernicus GmbH volume IV-4-W1. URL: http://www .isprs-ann-photogramm-remote-sens-spatial-inf-sci.net/ IV-4-W1/89/2016/. doi:10.5194/isprs-annals-IV-4-W1-89-2016.

Besuievsky, G., Barroso, S., Beckers, B., \& Patow, G. (2014). A Configurable LoD for Procedural Urban Models intended for Daylight Simulation. In G. Besuievsky, \& V. Tourre (Eds.), Eurographics Workshop on Urban Data Modelling and Visualisation. Strasbourg: The Eurographics Association. doi:10.2312/udmv. 20141073.

Biljecki, F., Heuvelink, G. B. M., Ledoux, H., \& Stoter, J. (2015a). Propagation of positional error in 3d GIS: estimation of the solar irradiation of building roofs. International Journal of Geographical Information Science, 29, 2269-2294. URL: http://dx.doi.org/10.1080/13658816.2015.1073292. doi:10.1080/13658816.2015.1073292.

Biljecki, F., Ledoux, H., \& Stoter, J. (2017). Does a Finer Level of Detail of a 3d City Model Bring an Improvement for Estimating Shadows? In A. AbdulRahman (Ed.), Advances in 3D Geoinformation Lecture Notes in Geoinformation and Cartography (pp. 31-47). Springer International Publishing. URL: http: //link. springer.com/chapter/10.1007/978-3-319-25691-7_2. doi:10.1007/ 978-3-319-25691-7_2.

Biljecki, F., Ledoux, H., Stoter, J., \& Zhao, J. (2014). Formalisation of the level of detail in 3d city modelling. Computers, Environment and Urban Systems, 48, 1-15. URL: http://www.sciencedirect.com/science/article/pii/ S0198971514000519. doi:10.1016/j.compenvurbsys. 2014.05.004.

Biljecki, F., Stoter, J., Ledoux, H., Zlatanova, S., \& Çöltekin, A. (2015b). Applications of 3d City Models: State of the Art Review. ISPRS International Journal of Geo-Information, 4, 2842-2889. URL: http://www.mdpi.com/2220-9964/4/ 4/2842. doi:10.3390/ijgi4042842.

Bremer, M., Mayr, A., Wichmann, V., Schmidtner, K., \& Rutzinger, M. (2016). A new multi-scale 3d-GIS-approach for the assessment and dissemination of solar income of digital city models. Computers, Environment and Urban Systems, 57, 144-154. URL: http://www.sciencedirect.com/science/article/ pii/S0198971516300151. doi:10.1016/j . compenvurbsys. 2016.02.007. 
Brito, M. C., Freitas, S., Guimarães, S., Catita, C., \& Redweik, P. (2017). The importance of facades for the solar PV potential of a Mediterranean city using LiDAR data. Renewable Energy, 111, 85-94. URL: http://www.sciencedirect. com/science/article/pii/S0960148117302768. doi:10.1016/j.renene. 2017. 03.085.

Casey, C., \& Mistrick, R. (2015). Simulation Tools for Architectural Daylighting and Integrated Controls (STADIC) - Utilities. URL: https: //www .radiance-online.org/community/workshops/2015-philadelphia/ presentations/day1/STADICUtilities-Radiance\%20Workshop2015.pdf.

Catita, C., Redweik, P., Pereira, J., \& Brito, M. C. (2014). Extending solar potential analysis in buildings to vertical facades. Computers $\&$ Geosciences, 66, 1-12. URL: http://www.sciencedirect.com/science/article/pii/ S0098300414000053. doi:10.1016/j.cageo.2014.01.002.

CEC (2018). California Energy Commission - Database of PV modules. URL: http://www.gosolarcalifornia.ca.gov/equipment/pv_modules.php.

Chung, T. J. (2002). Computational Fluid Dynamics. Cambridge University Press. doi:10.1017/CB09780511606205.

Compagnon, R. (2004). Solar and daylight availability in the urban fabric. Energy and Buildings, 36, 321-328. doi:10.1016/j . enbuild.2004.01.009.

Compagnon, R., \& Raydan, D. (2000). Irradiance and illuminance distributions in urban areas. In Architecture, City, Environment. Proceedings of PLEA 2000 (pp. 436-441). Cambridge, UK: James \& James (Science Publishers) Ltd. URL: https://multidoc.eia-fr.ch/record/228?ln=en.

Costanzo, V., Yao, R., Essah, E., Shao, L., Shahrestani, M., Oliveira, A. C., Araz, M., Hepbasli, A., \& Biyik, E. (2018). A method of strategic evaluation of energy performance of Building Integrated Photovoltaic in the urban context. Journal of Cleaner Production, 184, 82-91. URL: http://www.sciencedirect.com/science/ article/pii/S0959652618304517. doi:10.1016/j.jclepro.2018.02.139.

Fath, K., Stengel, J., Sprenger, W., Wilson, H. R., Schultmann, F., \& Kuhn, T. E. (2015). A method for predicting the economic potential of (building-integrated) photovoltaics in urban areas based on hourly Radiance simulations. Solar Energy, 116, 357-370. URL: http://www.sciencedirect.com/science/article/pii/ S0038092X15001413. doi:10.1016/j.solener.2015.03.023. 
Florio, P., Roecker, C., Probst, M. C. M., \& Scartezzini, J.-L. (2016). Visibility of Building Exposed Surfaces for the Potential Application of Solar Panels: A Photometric Model. The Eurographics Association. URL: https://diglib.eg. org:443/handle/10.2312/udmv20161419.

Fogl, M., \& Moudrý, V. (2016). Influence of vegetation canopies on solar potential in urban environments. Applied Geography, 66, 73-80. URL: http://www. sciencedirect.com/science/article/pii/S0143622815300175. doi:10.1016/ j.apgeog. 2015.11.011.

Freitas, S., Catita, C., Redweik, P., \& Brito, M. C. (2015). Modelling solar potential in the urban environment: State-of-the-art review. Renewable and Sustainable Energy Reviews, 41, 915-931. URL: http://www.sciencedirect.com/science/ article/pii/S1364032114007461. doi:10.1016/j.rser.2014.08.060.

Iaccarino, G. (2009). Quantification of uncertainty in flow simulations using probabilistic methods. Technical Report DTIC Document. URL: http://oai.dtic. mil/oai/oai?verb=getRecord\&metadataPref ix=html\&identifier=ADA568130.

IEA (2002). Potential for Building Integrated Photovoltaics. Full Technical Report PVPS T7- 4 International Energy Agency.

IEA (2013). Transition to Sustainable Buildings: Strategies and opportunities to 2050. Exceutive summary International Energy Agency Paris. URL: http:// www . iea.org/Textbase/npsum/building2013SUM. pdf.

IESNA (2012). IES LM-83-12 IES Spatial Daylight Autonomy (sDA) and Annual Sunlight Exposure (ASE). Technical Report IES LM-83-12 Illuminating Engineering Society of North America New York, NY, USA.

Jakubiec, J. A., \& Reinhart, C. F. (2013). A method for predicting city-wide electricity gains from photovoltaic panels based on LiDAR and GIS data combined with hourly Daysim simulations. Solar Energy, 93, 127-143. URL: http: //www . sciencedirect.com/science/article/pii/S0038092X13001291. doi:10.1016/ j.solener. 2013.03 .022 .

Kanters, J., Wall, M., \& Kjellsson, E. (2014). The Solar Map as a Knowledge Base for Solar Energy Use. Energy Procedia, 48, 1597-1606. URL: http://www. sciencedirect.com/science/article/pii/S1876610214004421. doi:10.1016/ j.egypro.2014.02.180. 
Kämpf, J. H. (2009). On the modelling and optimisation of urban energy fluxes, . URL: https://infoscience.epfl.ch/record/141956. doi:10.5075/ epfl-thesis-4548, urn:nbn:ch:bel-epfl-thesis4548-9.

Mardaljevic, J., \& Rylatt, M. (2000). An image-based analysis of solar radiation for urban settings. In Architecture, City, Environment. Proceedings of PLEA 2000 (pp. 442-447). Cambridge, UK: James \& James (Science Publishers) Ltd. URL: https://multidoc.eia-fr.ch/record/228?ln=en.

Montavon, M. (2010). Optimisation of Urban Form by the Evaluation of the Solar Potential. PhD thesis École Polytechnique Fédérale de Lausanne (EPFL) Lausanne. URL: http://infoscience.epfl.ch/record/145897.

Nault, E., Moonen, P., Rey, E., \& Andersen, M. (2017). Predictive models for assessing the passive solar and daylight potential of neighborhood designs: A comparative proof-of-concept study. Building and Environment, . URL: https://www . sciencedirect.com/science/article/pii/S0360132317300197. doi:10.1016/ j.buildenv.2017.01.018.

Nault, E., Rey, E., \& Andersen, M. (2013). Early design phase evaluation of urban solar potential: Insights from the analysis of six projects. In Proceedings of BS2013 (pp. 177-184). Chambéry, France. URL: http://infoscience.epfl.ch/record/ $187120 /$.

Niyogi, P., Chakrabartty, S. K., \& Laha, M. K. (2006). Introduction to Computational Fluid Dynamics. Pearson India. URL: http://proquest.safaribooksonline. com/book/electrical-engineering/computer-engineering/9788177587647/ firstchapter.

Nouvel, R., Zirak, M., Coors, V., \& Eicker, U. (2017). The influence of data quality on urban heating demand modeling using $3 \mathrm{~d}$ city models. Computers, Environment and Urban Systems, 64, 68-80. URL: http://www. sciencedirect.com/science/article/pii/S0198971516304306. doi:10.1016/ j.compenvurbsys. 2016.12.005.

Peronato, G. (2014). Built density, solar potential and daylighting: Application of parametric studies and performance simulation tools in urban design. MArch thesis Università Iuav di Venezia Venice. URL: https://infoscience.epfl.ch/ record/201758? ln=en. 
Peronato, G., Bonjour, S., Stoeckli, J., Rey, E., \& Andersen, M. (2016a). Sensitivity of calculated solar irradiation to the level of detail: insights from the simulation of four sample buildings in urban areas. In PLEA 2016 - Cities, Buildings, People: Towards Regenerative Environments, Proceedings of the 32nd International Conference on Passive and Low Energy Architecture;. Los Angeles volume 2.

Peronato, G., Rey, E., \& Andersen, M. (2015). Sampling of building surfaces towards an early assessment of BIPV potential in urban contexts. In Proceedings of PLEA2015 Architecture in (R)Evolution. Bologna. URL: http: //www . plea2015. it/book/download . php?id=642.

Peronato, G., Rey, E., \& Andersen, M. (2016b). 3d-modeling of vegetation from LiDAR point clouds and assessment of its impact on façade solar irradiation. In ISPRS - International Archives of the Photogrammetry, Remote Sensing and Spatial Information Sciences (pp. 67-70). Athens volume XLII-2/W2. doi:10.5194/isprs-archives-XLII-2-W2-67-2016.

Prada, A., Pernigotto, G., Baggio, P., Gasparella, A., \& Mahdavi, A. (2014). Effect of Solar Radiation Model on the Predicted Energy Performance of Buildings. In International High Performance Buildings Conference. URL: http://docs.lib. purdue.edu/ihpbc/130.

Rastogi, P. (2016). On the sensitivity of buildings to climate. PhD Thesis 895 Ecole polytechnique fédérale de Lausanne Lausanne, Switzerland. URL: https: //infoscience.epfl.ch/record/220971.

Reinhart, C. F., \& Walkenhorst, O. (2001). Validation of dynamic RADIANCEbased daylight simulations for a test office with external blinds. Energy and Buildings, 33, 683-697. URL: http://www.sciencedirect.com/science/article/ pii/S0378778801000585. doi:10.1016/S0378-7788(01)00058-5.

Rey, E., Lufkin, S., Ballif, C., Wuestenhagen, R., Wittkopf, S., \& Bacher, J.-P. (2015). Building integrated photovoltaics | ACTIVE INTERFACES.

Š́ri, M., \& Hofierka, J. (2004). A New GIS-based Solar Radiation Model and Its Application to Photovoltaic Assessments. Transactions in GIS, 8, 175-190. URL: http://onlinelibrary.wiley.com/doi/10.1111/j.1467-9671. 2004.00174.x/abstract. doi:10.1111/j.1467-9671.2004.00174.x.

Robinson, D., \& Stone, A. (2004a). Irradiation modelling made simple: the cumulative sky approach and its applications. In Proceedings of PLEA 2004. Eindhoven, The Netherlands. 
Ward-Larson, G., \& Shakespeare, R. (1998). Rendering with radiance: the art and science of lighting visualization. San Francisco, CA, USA: Morgan Kaufmann Publishers Inc. URL: http://portal . acm.org/citation. cfm?id=286090. 
Wate, P., Coors, V., Robinson, D., \& Iglesias, M. (2016). Qualitative screening method for impact assessment of uncertain building geometry on thermal energy demand predictions. ISPRS - International Archives of the Photogrammetry, Remote Sensing and Spatial Information Sciences, XLII-2/W2, 127-134. URL: http://www .int-arch-photogramm-remote-sens-spatial-inf-sci.net/ XLII-2-W2/127/2016/. doi:10.5194/isprs-archives-XLII-2-W2-127-2016.

${ }_{950}$ Wieland, M., Nichersu, A., Murshed, S. M., \& Wendel, J. (2015). Computing solar radiation on CityGML building data. In 18th AGILE international conference on geographic informaton science. 